\title{
Álgebras de Bernstein
}

\author{
William Vieira
}

\author{
DISSERTAÇÃO APRESENTADA \\ $\mathrm{AO}$ \\ INSTITUTO DE MATEMÁTICA E ESTATÍSTICA \\ DA \\ UNIVERSIDADE DE SÃO PAULO \\ PARA OBTENÇÃO DO GRAU \\ $\mathrm{DE}$ \\ MESTRE EM MATEMÁTICA \\ Área de Concentração: Álgebra \\ Orientador: Prof. Dr. Juan Carlos Gutiérrez Fernández
}

São Paulo, 14 de Outubro de 2005. 
Este exemplar corresponde à redação final da dissertação devidamente corrigida e defendida por William Vieira e aprovada pela comissão julgadora.

São Paulo, 14 de Outubro de 2005.

Banca examinadora:

Prof. Dr. Juan Carlos Gutiérrez Fernández (orientador) - IME - USP

Prof. Dr. Henrique Guzzo Jr. - IME - USP

Prof. Dr. Antonio José Engler - IMECC - UNICAMP 
Para os meus queridos pais, pelo amor e dedicação de toda vida. 


\section{Agradecimentos}

À Maria Rita, pelo apoio, incentivo e paciência, e à minha pequena Maluzinha, cujo brilho dos olhos e entusiasmo pela vida fizeram-me continuar.

Aos meus irmãos Gisele e Douglas, pela amizade e carinho.

Nesses quase 10 anos de IME felizmente fiz muitos amigos e, para não cometer a injustiça de esquecer alguém especial, deixo aqui meus sinceros agradecimentos e um grande abraço para todos aqueles que fizeram parte dessa caminhada.

Finalmente, agradeço ao meu orientador, professor Juan Carlos, não apenas pela grande ajuda, paciência e ensinamentos, mas também pela amizade e bom humor que marcaram nossas conversas.

Este trabalho também é dedicado a Ananda, Éric, Giovana, Gustavo, Karen, Kelly, Laura, Maria Luísa, Nicole e Sofia, os pequenos notáveis da minha familia. Que eles cresçam felizes e se apaixonem pela busca do conhecimento! 


\begin{abstract}
In this work we study the invariance of $P$-subspaces and the solubility of the barideal $N=U \oplus V$ in the Bernstein algebras. We also show some relations between these algebras and those originated by the genetic motivation, as the genetic, train and train principal algebras.
\end{abstract}




\section{Resumo}

Neste trabalho estudamos a invariância dos $P$-subespaços e a solubilidade do barideal $N=U \oplus V$ nas Álgebras de Bernstein. Apresentamos também algumas relações entre estas álgebras e aquelas surgidas pela motivação genética, como as álgebras genéticas, train e train principal. 


\section{Índice}

Introdução 1

1 Álgebras de Bernstein 3

1.1 Conceitos básicos . . . . . . . . . . . . . . 3

1.2 Álgebras de Bernstein-Jordan . . . . . . . . . . . . . . . 15

2 Invariância de $P$-subespaços $\quad 20$

2.1 Conceitos básicos . . . . . . . . . . . . . . . . . 21

$2.2 \quad$ P-subespaços . . . . . . . . . . . . . . . 23

2.3 Invariância de $P$-subespaços nas álgebras de Bernstein . . . . . 26

3 Álgebras de Bernstein e álgebras genéticas 38

3.1 Álgebras báricas: uma classificação . . . . . . . . . . . . . 38

3.2 Álgebras de Bernstein e outras álgebras genéticas . . . . . . . 48

4 Solubilidade do barideal $\quad 55$

4.1 Nilpotência e Solubilidade do barideal . . . . . . . . . . . 56 
Índice

ii

Referências Bibliográficas

71

Índice Remissivo

76 


\section{Introdução}

O uso de métodos matemáticos para representar as relações genéticas de uma população datam do ínicio do século XX, com a famosa Lei de HardyWeinberg, de 1908. Outros trabalhos foram desenvolvidos desde então, por exemplo por Fisher, Haldane e Wright (ver [26]). Em 1923, após a elaboração de uma síntese dos trabalhos desenvolvidos até sua época, S.Bernstein propôs - e resolveu parcialmente - o problema da caracterização de todos os operadores de evolução que satisfazem o princípio estacionário. Tal princípio é uma generalização das Leis de Mendel e do clássico teorema de Hardy-Weinberg.

Entre 1939 e 1941 I.M.H Etherington inaugura, com um seqüência de artigos, um novo campo de estudo da Matemática, as álgebras genéticas. Nesta série de trabalhos, Etherington dá uma formulação matemática precisa das Leis de Mendel e introduz conceitos fundamentais para esta teoria, como o de álgebras báricas, train e train especial. Desde então, inúmeros autores têm trabalhado com estas álgebras. Dentro deste grupo de autores, devemos destacar Schafer e Gonshor que durante a primeira metade da década de 50 introduzem o conceito de álgebra genética e demonstram teoremas funda- 
mentais sobre elementos idempotentes e potências plenas nas álgebras train especiais. Aproximadamente 50 anos após S.Bernstein ter formulado seu problema, Yuri I. Lyubich e Ph. Holgate, independentemente, definem as álgebras de Bernstein, dando uma formulação algébrica para o problema.

O objetivo central deste trabalho é o estudo da estrutura das álgebras de Bernstein, a invariância dos $P$-subespaços, a nilpotência e solubilidade do barideal e as relações entre estas álgebras e outras álgebras báricas. No Capítulo 1 introduzimos os conceitos básicos para o estudo desta teoria, como a Decomposição de Peirce e identidades básicas. Dedicamos ainda uma seção para alguns resultados elementares sobre as álgebras de Bernstein-Jordan, pois muitas das propriedades e resultados sobre as álgebras de Bernstein são obtidos a partir do estudo deste tipo de álgebras.

O Capítulo 2 traz o estudo da invariância dos $P$-subespaços. Os $P$ subespaços são, em linhas gerais, subespaços vetoriais de uma álgebra de Bernstein $A=K e \oplus U \oplus V$ que tem expressão polinomial em termos de $U$ e $V$, sendo estes últimos subespaços surgidos na decomposição de Peirce da álgebra de Bernstein.

No Capítulo 3 estudamos as relações entre as álgebras de Bernstein e outras álgebras báricas como as álgebras genéticas, train, train principal e train especial. Por fim, no Capítulo 4, estudamos a solubilidade do barideal $N$ de uma álgebra de Bernstein $A=K e \oplus N$. Mais precisamente, demonstramos que $\left(N^{2}\right)^{5}=0$ e $N^{[3]}=0$. 
CAPÍtulo 1

\section{Álgebras de Bernstein}

As álgebras que surgem na genética são, em geral, comutativas e não necessariamente associativas. Entre elas, as álgebras de Bernstein tem um papel relevante. Estas álgebras surgem em relação a um problema proposto por S.Bernstein em 1923 sobre as condições nas quais uma população "alcança" o equilíbrio após uma geração. Dentro das técnicas de estudo destas álgebras estão a análise dos elementos idempotentes e a Decomposição de Peirce associada a estes idempotentes.

Neste capítulo apresentamos uma série de definições e resultados básicos referentes a estas álgebras, introduzidas independentemente por Lyubich [25] e Holgate [21], e que serão a base para nosso trabalho.

\subsection{Conceitos básicos}

Seja $K$ um corpo de característica diferente de 2 e 3 . Uma álgebra sobre o corpo $K$ é um espaço vetorial $A$ sobre $K$ no qual está definida uma aplicação, 
chamada produto, de $A \times A$ em $A$ que satisfaz

$$
\begin{gathered}
x(y+z)=x y+x z, \\
(y+z) x=y x+z x, \\
\alpha(x y)=x(\alpha y),
\end{gathered}
$$

para quaisquer $x, y, z \in A$ e qualquer $\alpha \in K$. A álgebra $A$ é dita comutativa ou associativa se cumpre, respectivamente, $x y=y x$ e $(x y) z=x(y z)$, para todo $x, y, z \in A$.

Dado um subconjunto $S$ de uma álgebra $A$, indicamos por $\langle S\rangle$ o subespaço vetorial de $A$ gerado por $S$. Denominamos anulador de $S$ o conjunto definido por

$$
\operatorname{ann} S=\{x \in A \mid x y=0 \text { para todo } y \in S\} .
$$

Além disso, um subespaço vetorial $I$ de $A$ é dito um $i d e a l$ de $A$ se cumpre-se $A I, I A \subseteq I$. Uma álgebra $A$ é dita simples se $A^{2} \neq 0$ e os únicos ideais de $A$ são os triviais, isto é, $\{0\}$ e $A$.

No decorrer deste trabalho $A$ denotará uma álgebra comutativa, não necessariamente associativa, de dimensão finita sobre o corpo $K$.

Há várias maneiras de definir as potências de elementos e subespaços em uma álgebra não associativa. A seguir destacamos dois tipos de potências que apresentam significação genética.

Seja $x$ um elemento da álgebra $A$. As potências principais de $x$ são definidas por

$$
x^{1}=x \quad \text { e } \quad x^{k}=x x^{k-1}, \quad(k \geq 2) .
$$


Analogamente, definimos as potências principais de um subespaço vetorial $U$ de $A$ por

$$
U^{1}=U \quad \text { e } \quad U^{k}=U U^{k-1}, \quad(k \geq 2) .
$$

Por outro lado, as potências plenas de $x$ e $U$ são definidas, respectivamente, por

$$
\begin{array}{cccc}
x^{[0]}=x & \text { e } & x^{[k]}=x^{[k-1]} x^{[k-1]}, & (k \geq 1), \\
U^{[0]}=U & \text { e } & U^{[k]}=U^{[k-1]} U^{[k-1]}, & (k \geq 1) .
\end{array}
$$

Além disso, as potências de $U$ são definidas por

$$
U^{(1)}=U \quad \text { e } \quad U^{(k)}=\sum_{i+j=k} U^{(i)} U^{(j)}, \quad(k \geq 2) .
$$

Um elemento $x$ da álgebra $A$ é dito nilpotente (principal) se existe $r \in \mathbb{N}$ tal que $x^{r}=0$. Uma álgebra $A$ é dita nil se todos os seus elementos são nilpotentes.

Uma função $\omega: A \rightarrow K$ é um caracter de $A$ se $\omega$ é um homomorfismo não nulo de álgebras, isto é, $\omega(x+y)=\omega(x)+\omega(y), \omega(x y)=\omega(x) \omega(y)$ e $\omega(\alpha x)=\alpha \omega(x)$, para todo $x, y \in A, \alpha \in K$. Tal homomorfismo é chamado função peso. O par $(A, \omega)$ é chamado álgebra bárica, e denotaremos por $N$ o núcleo do homomorfismo $\omega$. Observamos que, se $I$ é um ideal de $A$ com $I \subseteq N$, então $\bar{\omega}: A / I \rightarrow K$, definido por $\bar{\omega}(x+I)=\omega(x)$, para todo $x \in A$, é um homomorfismo da álgebra quociente $A / I$ em $K$. Portanto, o $\operatorname{par}(A / I, \bar{\omega})$ também é uma álgebra bárica.

Definição 1 Uma álgebra bárica $(A, \omega)$ é dita uma álgebra de Bernstein quando vale a identidade

$$
\left(x^{2}\right)^{2}=\omega\left(x^{2}\right) x^{2}, \quad \text { para todo } x \in A .
$$


O lema seguinte é devido a Holgate [21].

Lema 1.1 Uma álgebra de Bernstein $(A, \omega)$ possui exatamente um homomorfismo de peso.

Demonstração. Pela definição de álgebra de Bernstein temos um homomorfismo não-trivial $\omega: A \rightarrow K$. Seja $\varphi: A \rightarrow K$ outro homomorfismo não-trivial. Desde que $\varphi$ é compatível com a multiplicação em $A$, a identidade (1.1.1) implica que $\varphi(y)^{4}=\varphi\left(\left(y^{2}\right)^{2}\right)=\varphi(0)=0$ para todo $y \in N$, logo $\varphi(y)=0$ para todo $y \in N$. Portanto $\operatorname{ker} \varphi=\operatorname{ker} \omega$, já que $\varphi$ é não nulo.

Agora seja $x \in A-N$. Então $\omega(x) \neq 0$ e $\varphi(x) \neq 0$ já que $\operatorname{ker} \varphi=$ ker $\omega$. Além disso, $\omega\left(\frac{x^{2}}{\omega(x)}-x\right)=0$, e assim $\frac{x^{2}}{\omega(x)}-x \in N$. Então segue que $0=\varphi\left(\frac{x^{2}}{\omega(x)}-x\right)=\frac{\varphi(x)^{2}}{\omega(x)}-\varphi(x)=\varphi(x)\left(\frac{\varphi(x)}{\omega(x)}-1\right)$ e como $\varphi(x) \neq 0$, temos que $\frac{\varphi(x)}{\omega(x)}-1=0$, isto é $\varphi(x)=\omega(x)$.

Amparados neste último resultado, passaremos a denotar o par $(A, \omega)$ apenas por $A$ a fim de simplificar a notação.

Lema 1.2 Seja A uma álgebra de Bernstein. Então

$$
2 x^{i} x^{j}=\omega\left(x^{i}\right) x^{j}+\omega\left(x^{j}\right) x^{i}
$$

para todo $x \in A$ com $i, j \geq 2$.

Demonstração. Linearizando (1.1.1) temos as identidades

$$
\begin{gathered}
2 x^{2}(x y)=\omega(x y) x^{2}+\omega\left(x^{2}\right) x y \\
4(x y)(x z)+2 x^{2}(y z)=\omega(y z) x^{2}+2 \omega(x y) x z+\omega\left(x^{2}\right) y z+2 \omega(x z) x y
\end{gathered}
$$


para todo $x, y, z \in A$. Agora, fazendo $y=x^{j-1} \mathrm{em}$ (1.1.3), temos

$$
2 x^{2}\left(x^{j}\right)=\omega\left(x^{j}\right) x^{2}+\omega\left(x^{2}\right) x^{j}
$$

para todo $j \geq 2$. Seja agora $2<r \leq s$ e assuma, por hipótese de indução, que a identidade (1.1.2) é verdadeira para todo par $(i, j)$ com $2 \leq i<r$ e $i \leq j$. Assim por hipótese de indução segue

$$
\begin{aligned}
2 x^{2}\left(x^{r-1} x^{s-1}\right) & =x^{2}\left\{\omega\left(x^{r-1}\right) x^{s-1}+\omega\left(x^{s-1}\right) x^{r-1}\right\} \\
& =\omega\left(x^{r-1}\right) x^{2} x^{s-1}+\omega\left(x^{s-1}\right) x^{2} x^{r-1} \\
& =\frac{1}{2}\left\{\omega\left(x^{r+1}\right) x^{s-1}+\omega\left(x^{r+s-2}\right) x^{2}+\omega\left(x^{s+1}\right) x^{r-1}+\omega\left(x^{r+s-2}\right) x^{2}\right\} \\
& =\omega\left(x^{r+s-2}\right) x^{2}+\frac{1}{2}\left\{\omega\left(x^{r+1}\right) x^{s-1}+\omega\left(x^{s+1}\right) x^{r-1}\right\} \\
& =\omega\left(x^{r+s-2}\right) x^{2}+\omega\left(x^{2}\right) x^{r-1} x^{s-1} .
\end{aligned}
$$

Agora, tomando $y=x^{r-1}$ e $z=x^{s-1}$ em (1.1.4) segue que

$$
\begin{gathered}
4\left(x^{r} x^{s}\right)+2 x^{2}\left(x^{r-1} x^{s-1}\right)=\omega\left(x^{r-1} x^{s-1}\right) x^{2}+2 \omega\left(x^{r}\right) x^{s} \\
+\omega\left(x^{2}\right) x^{r-1} x^{s-1}+2 \omega\left(x^{s}\right) x^{r}
\end{gathered}
$$

para todo $x \in A$, e combinado com a relação acima temos finalmente que

$$
2\left(x^{r} x^{s}\right)=\omega\left(x^{r}\right) x^{s}+\omega\left(x^{s}\right) x^{r}
$$

para todo $x \in A$.

Os próximos resultados caracterizam os elementos idempotentes de uma álgebra de Bernstein. Como veremos no decorrer deste trabalho, estes elementos tem um papel central no estudo da estrutura destas álgebras. Denotaremos por $e$ um idempotente não nulo de uma álgebra de Bernstein $A$, i.e. $e \neq 0$ e $e^{2}=e$. 
Lema 1.3 Seja A uma álgebra de Bernstein. O conjunto de idempotentes não nulos de $A$ é dado por

$$
I d A=\left\{x^{2} \mid x \in A, \quad \text { com } \omega(x)=1\right\} .
$$

Demonstração. Seja $e \in A$ um idempotente não nulo. Substituindo em (1.1.1) temos

$$
0=\left(e^{2}\right)^{2}-\omega^{2}(e) e^{2}=e-\omega\left(e^{2}\right) e=e-\omega(e) e .
$$

Logo, $\omega(e)=1$. Por outro lado, tome $x \in A \operatorname{com} \omega(x)=1$. Então $\omega\left(x^{2}\right)=$ $\omega^{2}(x)=1$ e portanto, de (1.1.1) segue que $\left(x^{2}\right)^{2}=x^{2}$ e assim $x^{2}$ é um idempotente de $A$.

Segue do lema anterior que toda álgebra de Bernstein possui, no mínimo, um idempotente $e$. Um elemento $x \in A$ é representado de maneira única como $x=\omega(x) e+y$, com $y \in N$. Substituindo está decomposição em (1.1.1), obtemos imediatamente as identidades abaixo

$$
\begin{aligned}
2 e(2 e y) & =2 e y \\
2 e y^{2}+(2 e y)^{2} & =y^{2}, \\
(2 e y) y^{2} & =0 \\
\left(y^{2}\right)^{2} & =0 .
\end{aligned}
$$

Como $N$ é um ideal de $A$, podemos definir o operador linear $M_{e}: N \rightarrow N$ por $M_{e}(y)=2 e y$, para $y \in N$. A identidade (1.1.5) garante que esse operador é uma projeção, isto é $M_{e}^{2}=M_{e}$. Assim, denotando por $U_{e}$ e $V_{e}$ a imagem e o núcleo de $M_{e}$, respectivamente, temos a decomposição

$$
N=U_{e} \oplus V_{e}
$$


onde

$$
U_{e}=\{x \in A \mid 2 e x=x\}, \quad V_{e}=\{x \in A \mid e x=0\}
$$

Segue ainda da identidade (1.1.8) as linearizações

$$
\begin{aligned}
x_{1}{ }^{2}\left(x_{2} x_{3}\right)+2\left(x_{1} x_{2}\right)\left(x_{1} x_{3}\right) & =0 \\
\left(x_{1} x_{2}\right)\left(x_{3} x_{4}\right)+\left(x_{1} x_{3}\right)\left(x_{2} x_{4}\right)+\left(x_{1} x_{4}\right)\left(x_{2} x_{3}\right) & =0
\end{aligned}
$$

para todo $x_{1}, x_{2}, x_{3}, x_{4} \in N$.

Lema 1.4 Para uma álgebra de Bernstein $A=K e \oplus U_{e} \oplus V_{e}$ temos as seguintes inclusões

$$
U_{e}^{2} \subseteq V_{e}, \quad V_{e}^{2} \subseteq U_{e}, \quad U_{e} V_{e} \subseteq U_{e}
$$

Demonstração. De (1.1.6) temos que $M_{e}\left(y^{2}\right)+M_{e}(y)^{2}=y^{2}$ para todo $y \in N$. Linearizando está igualdade temos que $M_{e}\left(y_{1} y_{2}\right)+M_{e}\left(y_{1}\right) M_{e}\left(y_{2}\right)=$ $y_{1} y_{2}$, para todo $y_{1}, y_{2} \in N$. Como $M_{e}(y)=y$ se e somente se $y \in U_{e}$, temos, tomando $y_{1}, y_{2} \in U_{e}$ na última equação, que $M_{e}\left(y_{1} y_{2}\right)+y_{1} y_{2}=y_{1} y_{2}$, de onde segue que $M_{e}\left(y_{1} y_{2}\right)=0$, portanto $y_{1} y_{2} \in V_{e}$ e assim, $U_{e}^{2} \subseteq V_{e}$.

Por outro lado, se $y_{2} \in V_{e}$, segue que $M_{e}\left(y_{1} y_{2}\right)=y_{1} y_{2}$, para todo $y_{1} \in N$. Desta forma, $N V_{e} \subseteq U_{e}$ e, em particular, $U_{e} V_{e} \subseteq U_{e}$ e $V_{e}^{2} \subseteq U_{e}$.

O próximo teorema caracteriza as álgebras de Bernstein através de identidades que relacionam $U_{e}$ e $V_{e}$ e serão fundamentais no desenvolvimento do nosso estudo.

Teorema 1.5 Em uma álgebra de Bernstein $A=K e \oplus U_{e} \oplus V_{e}$ valem as identidades

$$
u^{3}=u(u v)=u v^{2}=\left(u^{2}\right)^{2}=(u v)^{2}=0
$$


para quaisquer $u \in U_{e}$ e $v \in V_{e}$.

Reciprocamente, se $A=K e \oplus U_{e} \oplus V_{e}$ é uma álgebra bárica com e idempotente, eu $=\frac{1}{2} u, e v=0$ para todo $u \in U_{e}$ e $v \in V_{e}$, e valem (1.1.11) e (1.1.12), então A é uma álgebra de Bernstein.

Demonstração. Sejam $u \in U_{e}, v \in V_{e}$. Para cada $\alpha, \beta \in K$, substituindo $y$ por $\alpha u+\beta v$ em (1.1.7), temos

$$
\alpha^{3} u^{3}+2 \alpha^{2} \beta u(u v)+\alpha \beta^{2} u v^{2}=0
$$

Assim, para $\alpha=1$ e $\beta=0$ temos $u^{3}=0$ e agora, considerando $\alpha=\beta=1$, segue-se $u(u v)+u v^{2}=0$. Para $\alpha=2, \beta=1$ temos $4 u(u v)+u v^{2}=0$. Combinando as identidades anteriores obtemos que $u(u v)=0$ e $u v^{2}=0$.

Novamente, fazendo $y=\alpha u+\beta v$ em (1.1.8), temos $\alpha^{4}\left(u^{2}\right)^{2}+4 \alpha^{3} \beta u^{2}(u v)+2 \alpha^{2} \beta^{2} u^{2} v^{2}+4 \alpha^{2} \beta^{2}(u v)^{2}+4 \alpha \beta^{3}(u v) v^{2}+\beta^{4}\left(v^{2}\right)^{2}=0$

Já que a igualdade é válida para todo $\alpha, \beta \in K$, segue que

$$
u^{2} u^{2}=0, \quad u^{2}(u v)=0, \quad u^{2} v^{2}+2(u v)^{2}=0, \quad(u v) v^{2}=0, \quad\left(v^{2}\right)^{2}=0
$$

Finalmente, usando a linearização da identidade $x^{3}=0$, para todo $x \in U_{e}$ e observando que $v^{2} \in U_{e}$, segue-se que $u^{2} v^{2}=-2 u\left(u v^{2}\right)=0$. Assim, $(u v)^{2}=0$.

Reciprocamente, seja $A=K e \oplus U_{e} \oplus V_{e}$ uma álgebra bárica com $e$ idempotente, eu $=\frac{1}{2} u, e v=0$ para todo $u \in U_{e}$ e $v \in V_{e}$, e onde valem (1.1.11) e (1.1.12). Observamos primeiro que a álgebra $A$ é bárica com peso dado por $\omega(\lambda e+u+v)$ para cada $\lambda \in K, u \in U_{e}$ e $v \in V_{e}$. Vejamos agora que $A$ 
verifica as identidades $u^{2}(u v)=u^{2} v^{2}=v^{2}(u v)=\left(v^{2}\right)^{2}=0$ para todo $u \in U_{e}$ e $v \in V_{e}$. Linearizando $u^{3}=0$ temos

$$
u_{1}\left(u_{2} u_{3}\right)+u_{2}\left(u_{1} u_{3}\right)+u_{3}\left(u_{1} u_{2}\right)=0
$$

para todo $u_{1}, u_{2}, u_{3} \in U_{e}$. Agora, fazendo $u_{1}=u_{2}=u$ e $u_{3}=u v$, temos $2 u(u(u v))+(u v) u^{2}=0$, de onde vem que $(u v) u^{2}=0$. Fazendo $u_{1}=u_{2}=u$ e $u_{3}=v^{2}$ segue que $2 u\left(u v^{2}\right)+v^{2} u^{2}=0$, o que implica que $u^{2} v^{2}=0$. Por fim, $(u v) v^{2}=0$ e $\left(v^{2}\right)^{2}=0$ seguem da terceira identidade de (1.1.12), pois $U_{e} V_{e}, V_{e}^{2} \subseteq U_{e}$. Seja agora $x=\omega(x) e+u+v$ um elemento da álgebra. Então

$$
\begin{gathered}
x^{2}=\omega(x)^{2} e+2 \omega(x) u+2 u v+v^{2}+u^{2} \\
\left(x^{2}\right)^{2}=\omega(x)^{4} e+2 \omega(x)^{3} u+2 \omega(x)^{2}(u v)+\omega(x)^{2} v^{2}+\omega(x)^{2} u^{2}=\omega(x)^{2} x^{2} .
\end{gathered}
$$

Logo, $A$ é uma álgebra de Bernstein.

Se $A=K e \oplus U_{e} \oplus V_{e}$ é uma álgebra de Bernstein com idempotente $e$, então podemos decompor cada $x \in A$ da forma única $x=\omega(x) e+u+v$ com $u \in U_{e}$ e $v \in V_{e}$. Agora, segue do teorema anterior que

$$
x^{2}=\omega^{2}(x) e+\left(\omega(x) u+2 u v+v^{2}\right)+u^{2},
$$

$\operatorname{com} \omega(x) u+2 u v+v^{2} \in U_{e}$ e $u^{2} \in V_{e}$.

Linearizando as identidades de (1.1.12) temos

$$
\begin{array}{rlrl}
u\left(v_{1} v_{2}\right)=0, & u\left(u^{2} v\right)=0, & u^{2}\left(u^{2} v\right) & =0, \\
u_{1}\left(u_{2} v\right)+u_{2}\left(u_{1} v\right)=0, & u_{1}^{2}\left(u_{2} v\right)+2\left(u_{1} u_{2}\right)\left(u_{1} v\right) & =0, \\
u_{1}^{2}\left(u_{1} u_{2}\right)=0, & u_{1}^{2} u_{2}+2 u_{1}\left(u_{1} u_{2}\right)=0, \\
\left(u_{1} v\right)\left(u_{2} v\right)=0, \quad\left(u v_{1}\right)\left(u v_{2}\right) & =0, \\
\left(u_{1} u_{2}\right) v^{2}=u^{2}\left(v_{1} v_{2}\right) & =0 .
\end{array}
$$


Lema 1.6 Seja $e \in A$ um idempotente não nulo de uma álgebra de Bernstein. O conjunto dos idempotentes não nulos de $A$ é dado por

$$
I d A=\left\{e+u+u^{2} \mid u \in U_{e}\right\}
$$

Demonstração. Se $f=e+u+u^{2}$, com $u \in U$, então

$$
f^{2}=e+2 e u+u^{2}+2 u^{3}+\left(u^{2}\right)^{2}=e+u+u^{2}=f
$$

já que $\left(u^{2}\right)^{2}=0, u^{3}=0$ e $2 e u=u$.

Por outro lado, seja $f=e+u+v \in I d A$. Então temos $f^{2}=e+u+$ $2 u v+v^{2}+u^{2}=e+u+v$, de onde segue que $v=u^{2}$.

Sejam $e, f \in I d A$. Pelo lema anterior existe $\mathbf{u} \in U_{e}$ tal que $f=e+\mathbf{u}+\mathbf{u}^{2}$. O seguinte lema relaciona os subespaços $U_{e}, U_{f}$ e $V_{e}, V_{f}$.

Lema 1.7 Com as notações acima as aplicações lineares $\varphi_{\mathbf{u}}: U_{e} \rightarrow U_{f} e$ $\sigma_{\mathbf{u}}: V_{e} \rightarrow V_{f}$ definidas por

$$
\varphi_{\mathbf{u}}(u)=u+2 \mathbf{u} u, \quad \sigma_{\mathbf{u}}(v)=v-2\left(\mathbf{u}+\mathbf{u}^{2}\right) v
$$

para todo $u \in U_{e}$ e $v \in V_{e}$, são isomorfismos de espaços vetoriais.

Demonstração. Vamos mostrar inicialmente que $\varphi$ e $\sigma$ estão bem definidas. Sejam $u \in U_{e}$ e $v \in V_{e}$. Temos então, usando as identidades (1.1.15) a (1.1.19), que $2 f(u+2 \mathbf{u} u)=2\left(e+\mathbf{u}+\mathbf{u}^{2}\right)(u+2 \mathbf{u} u)=2 e u+4 e(\mathbf{u} u)+2 \mathbf{u} u+$ $4 \mathbf{u}(\mathbf{u} u)+2 \mathbf{u}^{2} u+2 \mathbf{u}^{2}(\mathbf{u} u)=u+2 \mathbf{u} u$. E também que $f\left(v-2 \mathbf{u} v-2 \mathbf{u}^{2}\right)=$ $\left(e+\mathbf{u}+\mathbf{u}^{2}\right)\left(v-2 \mathbf{u} v-2 \mathbf{u}^{2} v\right)=e v-2 e(\mathbf{u} v)-2 e\left(\mathbf{u}^{2} v\right)+\mathbf{u} v-2 \mathbf{u}(\mathbf{u} v)-$ $2 \mathbf{u}\left(\mathbf{u}^{2} v\right)+\mathbf{u}^{2} v-2 \mathbf{u}^{2} 2(\mathbf{u} v)-2 \mathbf{u}^{2}\left(\mathbf{u}^{2} v\right)=0$.

Seja agora $u \in \operatorname{ker} \varphi$. Temos então que $\varphi(u)=u+2 \mathbf{u} u=0$, donde $u=-2 \mathbf{u} u \in U_{e} \cap V_{e}=\{0\}$, e $\varphi$ é injetora. De maneira análoga, seja 
$v \in \operatorname{ker} \sigma$. Segue então que $\sigma(v)=v-2 \mathbf{u} v-2 \mathbf{u}^{2} v=0$, e $v=2 \mathbf{u} v+2 \mathbf{u}^{2} v \in$ $U_{e} \cap V_{e}=\{0\}, \operatorname{logo} \sigma$ também é injetora. Desta forma, $\operatorname{dim} U_{e} \leq \operatorname{dim} U_{f}$ e $\operatorname{dim} V_{e} \leq \operatorname{dim} V_{f}$. Como $\operatorname{dim} U_{e}+\operatorname{dim} V_{e}=\operatorname{dim} U_{f}+\operatorname{dim} V_{f}=\operatorname{dim} N$ e o barideal $N$ tem dimensão finita, temos que $\varphi$ e $\sigma$ são isomorfismos de espaços vetoriais.

A decomposição $A=K e \oplus U_{e} \oplus V_{e}$ denomina-se Decomposição de Peirce da álgebra $A$ relativa ao idempotente $e$. Do último lema resulta que as dimensões de $U_{e}$ e $V_{e}$ independem da escolha do idempotente. Disso decorre que o par $\left(1+\operatorname{dim} U_{e}, \operatorname{dim} V_{e}\right)$ é um invariante das álgebras de Bernstein de dimensão finita e é denominado o tipo da álgebra $A$.

Daremos a seguir a caracterização de algumas classes especiais de álgebras de Bernstein.

Teorema 1.8 Seja $A=K e \oplus U_{e} \oplus V_{e}$ uma álgebra de Bernstein decomposta com relação a um idempotente $e$.

I. As seguintes afirmações são equivalentes e, quando valem, A é chamada regular:

(a) $x^{2} y=\omega(x) x y$ para todo $x, y \in A$;

(b) $U_{e} V_{e}+V_{e}^{2}=0$ (i.e. $u v=0 e v^{2}=0$ para todo $u \in U_{e} e v \in V_{e}$ );

(c) O quadrado de todo $x=\omega(x) e+u+v \in A$ de peso 1 é dado por

$$
x^{2}=\omega^{2}(x) e+\omega(x) u+u^{2} .
$$

II. As seguintes afirmações são equivalentes e, quando valem, $A$ é chamada de excepcional:

(a) $U_{e}^{2}=0$ (i.e. $u^{2}=0$ para todo $u \in U_{e}$ );

(b) Para $x=\omega(x) e+u+v \in A$, temos $x=\omega^{2}(x) e+\omega(x) u+2 u v+v^{2}$. 
Demonstração. I. (a) $\rightarrow$ (b) Sejam $u \in U_{e}$ e $v \in V_{e}$. Então, para cada $\alpha, \beta \in K$, considerando $x=\alpha u+\beta v$ e $y=e$ na identidade (a), temos $0=\omega(x) x y=x^{2} y=\left[\left(2 \alpha \beta u v+\beta^{2} v^{2}\right)+\alpha^{2} u^{2}\right] e=\alpha \beta u v+\frac{\beta^{2}}{2} v^{2}$. Já que esta relação é válida para todo $\alpha, \beta \in K$, temos que $u v=0$ e $v^{2}=0$, donde segue $U_{e} V_{e}=0$ e $V_{e}^{2}=0$.

(b) $\rightarrow$ (c) É imediata.

(c) $\rightarrow$ (b) Sejam $u \in U, v \in V$. Para cada $\alpha, \beta \in K$ temos, segundo (c), que $e+\alpha u+\alpha^{2} u^{2}=(e+\alpha u+\beta v)^{2}=e+\left(\alpha u+2 \alpha \beta u v+\beta^{2} v\right)+\alpha^{2} u^{2}$, logo $2 \alpha \beta u v+\beta^{2} v^{2}=0$. Para $\alpha=0$ e $\beta=1$ temos que $v^{2}=0$ e agora, para $\alpha=0$ e $\beta=1$ segue que $u v=0$.

(b) $\rightarrow$ (a) Seja $x=\omega(x) e+u+v$ um elemento arbitrário de $A$. Então, pelo item (c) temos que $x^{2}=\omega^{2}(x)+\omega(x) u+u^{2}$, logo

$$
x^{2}-\omega(x) x=u^{2}-\omega(x) v \in V_{e}
$$

e assim,

$$
x^{2} y-\omega(x) x y=\left(x^{2}-\omega(x) x\right) y \in V_{e} A=0,
$$

para cada $y \in A$.

II. (a) $\leftrightarrow$ (b) É imediato a partir da relação (1.1.14).

Definição 2 Uma álgebra de Bernstein $A$ é dita nuclear quando $A=A^{2}$.

Notemos que, dada uma álgebra de Bernstein $A=K e \oplus U_{e} \oplus V_{e}$ temos $A^{2}=K e+U_{e}+U_{e} V_{e}+V_{e}^{2}+U_{e}^{2}=K e+U_{e}+U_{e}{ }^{2}$. Portanto, temos o seguinte resultado.

Lema 1.9 Seja $A=K e \oplus U_{e} \oplus V_{e}$ uma álgebra de Bernstein decomposta com relação a um idempontente e. Então $A$ é nuclear se, e somente se $U_{e}{ }^{2}=V_{e}$. 


\section{2 Álgebras de Bernstein-Jordan}

As álgebras de Bernstein-Jordan surgem naturalmente como quociente de álgebras de Bernstein. Isto constitui um fato relevante e é o motivo pelo qual dispensamos uma parte deste trabalho ao seu estudo. Uma álgebra comutativa $A$ é uma álgebra de Jordan se vale a identidade

$$
\left(x^{2} y\right) x=x^{2}(y x)
$$

para todo $x, y \in A$. Dizemos que uma álgebra de Bernstein $A$ é uma álgebra de Bernstein-Jordan se $A$ for uma álgebra de Jordan. E uma álgebra $A$ é de potências associativas se as potências principais de todo elemento $x$ de $A$ satisfazem a relação $x^{i} x^{j}=x^{i+j}$, para $i, j \geq 1$.

Em [24], Lyubich pela primeira vez relaciona as álgebras de Bernstein com as álgebras de Jordan e de potências associativas. Além disso, em 1977, Lyubich prova que toda álgebra de Bernstein-Jordan é de potências associativas e verifica a identidade train $x^{3}=\omega(x) x^{2}$. Posteriormente outros autores (González e Martínez [14] e Walcher [34]) estabelecem novas caracterizações para as Álgebras de Bernstein-Jordan.

Proposição 1.10 Seja A uma álgebra de Bernstein. São equivalentes:
(a) A é uma álgebra de Jordan;
(b) A é de potências associativas;
(c) $V_{e}^{2}=0$ para todo $e \in I d A$;
(d) Para todo e tem-se $V_{e}^{2}=0$ e $(u v) v=0$ para todo $u \in U_{e}$ e $v \in V_{e}$;
(e) Para algum e tem-se $V_{e}^{2}=0$ e $(u v) v=0$ para todo $u \in U_{e}$ e $v \in V_{e}$;
(f) A satisfaz a identidade $x^{3}=\omega(x) x^{2}$. 
Demonstração. (a) $\rightarrow$ (b) É conhecido que toda álgebra de Jordan é de potências associativas.

(b) $\rightarrow$ (c) Sejam $e$ idempotente e $v \in V_{e}$. Então para cada $\lambda \in K$ consideremos o elemento $x=e+\lambda v$. Temos que $x^{2}=e+\lambda^{2} v^{2}, x^{3}=$ $e+\frac{1}{2} \lambda^{2} v^{2}+\lambda^{3} v^{3}, x^{4}=e+\frac{1}{4} \lambda^{2} v^{2}+\lambda^{3} v^{3}+\lambda^{4} v^{4}$ e $x^{2} x^{2}=e+\lambda^{2} v^{2}+\lambda^{4} v^{4}$. Como $x^{4}=x^{2} x^{2}$, vem que $-\frac{3}{4} \lambda^{2} v^{2}+\lambda^{3} v^{3}=0$, para todo $\lambda \in K$. Portanto $v^{2}=0$ e assim, $V_{e}^{2}=0$.

(c) $\rightarrow$ (d) Sejam $e \in I d A, u_{0} \in U_{e}$ e $v_{0} \in V_{e}$. Considere o idempotente $f \in I d A$ dado por $f=e+u_{0}+u_{0}^{2}$. Das hipóteses e do Lema 1.7 temos que $V_{f}=\left\{v-2\left(u_{0}+u_{0}^{2}\right) v \mid v \in V_{e}\right\}=\left\{v-2 u_{0} v \mid v \in V_{e}\right\}$. Assim, $0=\left(v_{0}-2 u_{0} v_{0}\right)^{2}=v_{0}^{2}-4\left(u_{0} v_{0}\right) v_{0}+4\left(u_{0} v_{0}\right)^{2}=-4\left(u_{0} v_{0}\right) v_{0}$. Portanto, $\left(u_{0} v_{0}\right) v_{0}=0$ para quaisquer $e \in I d A, u_{0} \in U_{e}$ e $v_{0} \in V_{e}$.

$(\mathrm{d}) \rightarrow$ (e) É imediato.

(e) $\rightarrow$ (f) Seja $x=\alpha e+u+v \in A$, com $\alpha \in K, u \in U_{e}$ e $v \in V_{e}$. Substituindo em $x^{3}$ e $\omega(x) x^{2}$ temos que

$$
(\alpha e+u+v)^{2}=\alpha^{2} e+\alpha u+2 u v+v^{2}+u^{2},
$$

e

$$
\begin{aligned}
(\alpha e+u+v)^{3}= & \alpha^{3} e+\alpha^{2} e u+2 \alpha e(u v)+\alpha e v^{2}+\alpha e u^{2}+\alpha^{2} e u+\alpha u^{2}+ \\
& 2 u(u v)+u v^{2}+u^{3}+\alpha^{2} e v+\alpha u v+2(u v) v+v^{3}+u^{2} v \\
= & \alpha^{3} e+\alpha^{2} u+2 \alpha u v+\alpha v^{2}+\alpha u^{2} .
\end{aligned}
$$

Portanto, para qualquer $x \in A, x^{3}=\omega(x) x^{2}$.

(f) $\rightarrow$ (a) Linearizando $x^{3}=\omega(x) x^{2}$, temos

$$
x^{2} y+2(x y) x=\omega(y) x^{2}+2 \omega(x) x y .
$$


Multiplicando (1.2.2) por $x$ vem que

$$
\left(x^{2} y\right) x+2((x y) x) x=\omega(y) x^{3}+2 \omega(x)(x y) x,
$$

e substituindo $y=x y$ em (1.2.2) temos

$$
x^{2}(x y)+2(x(x y)) x=\omega(x) \omega(y) x^{2}+2 \omega(x)(x y) x .
$$

Finalmente, subtraindo (1.2.3) de (1.2.4) segue que

$$
x\left(x^{2} y\right)-x^{2}(x y)-\omega(y)\left(x^{3}-\omega(x) x^{2}\right)=0,
$$

donde $x\left(x^{2} y\right)=x^{2}(x y)$.

Desta última proposição segue uma maneira de caracterizar os ideais $I$ de uma álgebra de Bernstein $A$ cujo quociente $A / I$ é uma álgebra de Jordan.

Corolário 1.11 Sejam A uma álgebra de Bernstein e $I \subseteq N$ um ideal de A. Uma condição necessária e suficiente para que $A / I$ seja uma álgebra de Jordan é que $V_{e}^{2} \subseteq$ I para cada idempotente e de $A$.

Corolário 1.12 Seja A uma álgebra de Bernstein-Jordan. Então

$$
\left(x_{1} x_{2}\right) x_{3}+\left(x_{2} x_{3}\right) x_{1}+\left(x_{1} x_{3}\right) x_{2}=0
$$

para todo $x_{1}, x_{2}, x_{3} \in N$.

Corolário 1.13 Em uma álgebra de Bernstein-Jordan $A=K e \oplus U_{e} \oplus V_{e}$ temos

$$
v_{1} v_{2}=0, \quad\left(u v_{1}\right) v_{2}+\left(u v_{2}\right) v_{1}=0
$$

para todo $u \in U_{e}$ e $v_{1}, v_{2} \in V_{e}$. 
A proposição seguinte define o subespaço $L$ como a intersecção entre os subespaços $U_{e}$, quando $e$ percorre todos os idempotentes, e ainda que ele é um ideal da álgebra de Bernstein $A$. Este ideal foi introduzido por Lyubich (ver [26] página 96).

Proposição 1.14 Seja A uma álgebra de Bernstein. Então $L:=\bigcap_{e \in I d A} U_{e} e ́$ um ideal de $A$ e cumpre-se $L=U_{e} \cap a n n U_{e}$.

Demonstração. Seja $e$ um idempotente fixado de $A$. Vejamos que se verifica a igualdade $L=U_{e} \cap \operatorname{ann} U_{e}$. Seja $u \in L$. Para cada $x \in U_{e}, f=e+x+x^{2} \in$ $I d A$. Já que $u \in U_{f}$, existe $\bar{u} \in U_{e}$ tal que $u=\bar{u}+2 x \bar{u}$. Então $u-\bar{u}=2 x \bar{u} \in$ $U_{e} \cap U_{e}^{2} \subseteq U_{e} \cap V_{e}=0$, logo $u x=0$. Já que $x$ era um elemento arbitrário de $U_{e}$, segue-se $u U_{e}=0$. Portanto provamos que $L \subset U_{e} \cap \operatorname{ann} U_{e}$.

Por outro lado, se $u \in U_{e} \cap \operatorname{ann} U_{e}$, então $u=u+2 u x \in U_{e+x+x^{2}}$ para todo $x \in U_{e}$. Portanto $u \in \bigcap_{e \in I d A} U_{e}=L$ e assim está provada a outra inclusão.

Provaremos agora que $L$ é um ideal. Já que $L \subset U_{e}$ segue $e L \subset L$ e também $L U_{e} \subseteq\left(\operatorname{ann} U_{e}\right) U_{e}=0 \subset L$. Para todo $v \in V_{e}, \bar{u} \in L$ e $u \in U_{e}$ temos, por (1.1.16), que $0=\bar{u}(u v)+u(\bar{u} v)=u(\bar{u} v)$, de onde segue que $\left(L V_{e}\right) U_{e}=0$. Como $L V_{e} \subseteq U_{e}$ e $\left(L V_{e}\right) U_{e}=0$, segue que $L V_{e} \subseteq U_{e} \cap \operatorname{ann} U_{e}=L$ e $\operatorname{assim} L$ é um ideal de $A$.

Corolário 1.15 Seja A uma álgebra de Bernstein. Então A/L é uma álgebra de Bernstein-Jordan.

Demonstração. Pela proposição acima temos que $L$ é um ideal de $A$ e de (1.1.15) vem que $V_{e}^{2} \subseteq L$. Da Proposição 1.10 segue que $A / L$ é uma álgebra de Bernstein-Jordan. 
A seguir demonstramos algumas identidades que serão úteis no decorrer deste trabalho.

Lema 1.16 Sejam $A=K e \oplus U_{e} \oplus V_{e}$ uma álgebra de Bernstein, $u \in U_{e} e$ $v \in V_{e}$. Então temos, para $k \geq 1$, que:

(a) $u v^{k+1}=0$;

(b) $u L_{v}^{k}(u)=0$;

(c) $u L_{v}^{k}\left(u^{2}\right)=0$.

Demonstração. Para o item (a) observe que $v^{2} \in L$ e, como $L$ é um ideal, $v^{k+1} \in L$. Assim, $u v^{k+1}=0$.

No item (b), para $k=1$ temos $u(u v)=0$ pela identidade (1.1.12). Considere então uma álgebra quociente $\bar{A}=A / L$. Pelo Corolário $1.15, A / L$ é uma álgebra de Bernstein-Jordan e, pela Proposição 1.10, $(\bar{u} \bar{v}) \bar{v}=0$, para todo $\bar{u} \in \bar{U}_{\bar{e}}$ e $\bar{v} \in \bar{V}_{\bar{e}}$. Como $(u v) v \in L$ segue que $\left.L_{v}^{k-1}(u)=((\ldots(u v) v) \ldots) v\right)=$ $\overline{\bar{u}} \in L$ e assim temos que $u L_{v}^{k-1}=u \overline{\bar{u}}=0$.

No item (c) temos que $u^{2} v \in V_{e}^{2} \subset L, \operatorname{logo} L_{v}^{k}\left(u^{2}\right) \in L$ para todo $k \geq 1 \mathrm{e}$ $u L_{v}^{k}\left(u^{2}\right) \in u L=0$. 
CApÍtulo 2

\section{Invariância de $P$-subespaços}

Neste capítulo fazemos um estudo da invariância dos $P$-subespaços nas álgebras de Bernstein. Os $P$-subespaços são subespaços vetoriais de uma álgebra de Bernstein $A$ que tem expressão polinomial em termos de $U_{e}$ e $V_{e}$, subespaços de $A$. A princípio, cada $P$-subespaço está associado à decomposição da álgebra, relativa a um idempontente. Porém, conforme os estudos de R.A.Oliveira [28], M.L. Lelis [23] e J. Picanço [6] e [30], verificamos que alguns destes subespaços não dependem da escolha do idempotente, ou seja, são invariantes da álgebra $A$. A partir dos trabalhos de J. Picanço definimos as álgebras de Bernstein-Picanço como uma subclasse das álgebras de Bernstein para as quais todo $P$-subespaço tem dimensão invariante sobre a mudança do idempotente. 


\subsection{Conceitos básicos}

Sejam $A$ uma álgebra e $X, Y$ subespaços de $A$. Definimos os subespaços $X+Y$ e $X Y$ por

$$
\begin{gathered}
X+Y=\langle x+y \mid x \in X, y \in Y\rangle, \\
X Y=\langle x y \mid x \in X, y \in Y\rangle .
\end{gathered}
$$

Se $\mathcal{X}$ é o conjunto de geradores de $X$ e $\mathcal{Y}$ é o conjunto de geradores de $\mathrm{Y}$, então $X Y=\langle x y \mid x \in \mathcal{X}, y \in \mathcal{Y}\rangle$.

Observe ainda que, se $X, Y$ e $Z$ são subespaços de uma álgebra $A$, então $X(Y+Z)=X Y+X Z$, pois $x(y+z)=x y+x z$ e $x_{1} y+x_{2} z=x_{1}(y+0)+$ $x_{2}(0+z)$ para todo $x, x_{1}, x_{2} \in X, y \in Y$ e $z \in Z$.

Lema 2.1 Seja X um subespaço de uma álgebra comutativa A. Então:

(a) $X^{2}=\left\langle x^{2} \mid x \in X\right\rangle$;

(b) $X+X^{2}=\left\langle x+x^{2} \mid x \in X\right\rangle$.

Demonstração. (a) Basta observar que $x_{1} x_{2}=\frac{1}{2}\left(\left(x_{1}+x_{2}\right)^{2}-x_{1}^{2}-x_{2}^{2}\right)$ com $x_{1}, x_{2} \in X$. O item (b) segue de (a) e das identidades $x^{2}=\frac{1}{2}\left(x+x^{2}\right)+$ $\frac{1}{2}\left(-x+(-x)^{2}\right)$ e $x=\left(x+x^{2}\right)-x^{2}$.

Dada uma família $\left\{X_{n}\right\}_{n \in I}$ de subespaços de uma álgebra, a soma e a intersecção de elementos dessa família produz dois novos subespaços. Demonstraremos algumas igualdades para as famílias $\left\{U_{f}\right\}_{f \in I d A}$ e $\left\{V_{f}\right\}_{f \in I d A}$. 
Proposição 2.2 Em uma álgebra de Bernstein $A=K e \oplus U_{e} \oplus V_{e}$ valem as seguintes igualdades:

(a) $\bigcap_{f \in I d A} V_{f}=V_{e} \cap \operatorname{ann}\left(U_{e} \oplus U_{e}^{2}\right)$;

(b) $\sum_{f \in I d A} U_{f}=U_{e} \oplus U_{e}^{2}$;

(c) $\sum_{f \in I d A} V_{f}=\left(U_{e} V_{e}+U_{e}^{2} V_{e}\right) \oplus V_{e}$.

Demonstração. Para o item (a) seja $w \in \bigcap_{f \in I d A} V_{f}$, então, para cada $u \in U_{e}$, existe $v \in V_{e}$ tal que $w=v-2\left(u+u^{2}\right) v$. Como, particularmente, $w \in V_{e}$, segue que $v-w=2\left(u+u^{2}\right) v \in U_{e} \cap V_{e}=0$, logo $w=v$ e $\left(u+u^{2}\right) v=0$ para todo $u \in U_{e}$. Do Lema 2.1 temos que $w \in V_{e} \cap \operatorname{ann}\left(U_{e} \oplus U_{e}^{2}\right)$ e assim, $\bigcap_{f \in I d A} V_{f} \subseteq V_{e} \cap \operatorname{ann}\left(U_{e} \oplus U_{e}^{2}\right)$. Agora, seja $v \in V_{e} \cap \operatorname{ann}\left(U_{e} \oplus U_{e}^{2}\right)$. Então, para todo $u \in U_{e}, v=v-2\left(u+u^{2}\right) v \in V_{f}$, logo $v \in \bigcap_{f \in I d A} V_{f}$, o que conclui a igualdade.

No item (b), usando os Lemas 2.1 e 1.7 segue que $\sum_{f \in I d A} U_{f}=\langle x+$ $2 u x\left|x, u \in U_{e}\right\rangle \subseteq U_{e} \oplus U_{e}^{2}$. Por outro lado temos obviamente que $U_{e} \subset$ $\sum_{f \in I d A} U_{f}$, e também $u x=\frac{1}{2}(x+2 u x)-\frac{1}{2} x \in \sum_{f \in I d A} U_{f}$. Portanto, a igualdade está demonstrada.

Para o item (c), como para cada idempotente $f=e+u+u^{2}$ cumpre-se $V_{f}=\left\{v-2\left(u+u^{2}\right) v \mid v \in V_{e}\right\}$, temos $\sum_{f \in I d A} V_{f}=\left\langle v-2\left(u+u^{2}\right) v\right| u \in$ $\left.U_{e}, v \in V_{e}\right\rangle$. Por outro lado, como $V_{e} \subset \sum_{f \in I d A} V_{f}$, pelo Lema 2.1 temos $\sum_{f \in I d A} V_{f}=V_{e}+\left\langle\left(u+u^{2}\right) \mid u \in U_{e}\right\rangle V_{e}=V_{e} \oplus\left(U_{e}+U_{e}^{2}\right) V_{e}=V_{e} \oplus\left(U_{e} V_{e}+U_{e}{ }^{2} V_{e}\right)$, o que verifica a igualdade. 
Definindo

$$
\widetilde{U}=\sum_{f \in I d A} U_{f} \quad \text { e } \quad \tilde{V}=\sum_{f \in I d A} V_{f}
$$

temos, da proposição anterior, que $\widetilde{U}=U_{e} \oplus U_{e}{ }^{2}$ e $\widetilde{V}=\left(U_{e} V_{e}+U_{e}{ }^{2} V_{e}\right) \oplus V_{e}$.

Ainda sobre a última proposição é importante observar que os subespaços $\widetilde{U}$ e $\tilde{V}$ não dependem da escolha do idempotente.

Na seção seguinte definimos o conceito de $P$-subespaço e destacamos algumas propriedades que serão importantes para o estudo da invariância desses elementos.

\section{$2.2 \quad P$-subespaços}

Seja $A=K e \oplus U_{e} \oplus V_{e}$ a decomposição de Peirce de uma álgebra de Bernstein em relação a um idempotente e. Definimos recursivamente os subespaços monomiais de $A$ determinados pelos subespaços $U_{e}$ e $V_{e}$ da seguinte forma:

(1) $U_{e}$ e $V_{e}$ são subespaços monomiais determinados por $U_{e}$ e $V_{e}$;

(2) Se $m_{1}$ e $m_{2}$ são subespaços monomiais determinados por $U_{e}$ e $V_{e}$, então $m=m_{1} m_{2}$ é um subespaço monomial determinado por $U_{e}$ e $V_{e}$.

Os seguintes subespaços são exemplos de subespaços monomiais determinados por $U_{e}$ e $V_{e}$ :

$$
\begin{aligned}
U_{e} V_{e}^{2} & \left.=\left\langle u\left(v_{1} v_{2}\right)\right| u \in U_{e} \text { e } v_{1}, v_{2} \in V_{e}\right\rangle \\
\left(U_{e} V_{e}\right) V_{e} & \left.=\left\langle\left(u v_{1}\right) v_{2}\right| u \in U_{e} \text { e } v_{1}, v_{2} \in V_{e}\right\rangle \\
V_{e}^{3} & =\left\langle\left(v_{1} v_{2}\right) v_{3} \mid v_{1}, v_{2}, v_{3} \in V_{e}\right\rangle \\
\left(U_{e} V_{e}\right)^{2} & \left.=\left\langle\left(u_{1} v_{1}\right)\left(u_{2} v_{2}\right)\right| u_{1}, u_{2} \in U_{e} \text { e } v_{1}, v_{2} \in V_{e}\right\rangle
\end{aligned}
$$


Chamamos de subespaço polinomial de $A$ determinado por $U_{e}$ e $V_{e}$ aos subespaços de $A$ que são somas finitas de subespaços monomiais determinados por $U_{e}$ e $V_{e}$.

Seja $K[U, V]$ o anel de polinômios livre nas variáveis comutativas e não associativas $U$ e $V$, e seja $\mathcal{P} \subseteq K[U, V]$ o subconjunto formado pelo polinômio 0 e pelos polinômios com todos os coeficientes iguais a 1 e o termo constante igual a 0 . Para cada elemento $p(U, V)$ de $\mathcal{P}$, e cada idempotente $e$ de $A$, corresponde um subespaço polinomial determinado ao substituir $U$ e $V$ por $U_{e}$ e $V_{e}$, respectivamente. Tal subespaço polinomial assim determinado é chamado $P$-subespaço e será denotado por $p\left(U_{e}, V_{e}\right)$ ou simplesmente $p_{e}$. Denotaremos por $\partial p(U, V)$ o grau do polinômio $p(U, V) \in \mathcal{P}$.

Definiremos recursivamente os monômios pares e ímpares de $K[U, V]$ da seguinte forma:

(1) $U$ é par e $V$ é ímpar;

(2) Se $m_{1}(U, V)$ e $m_{2}(U, V)$ são pares e $n_{1}(U, V)$ e $n_{2}(U, V)$ são ímpares, então $m_{1}(U, V) n_{1}(U, V)$ e $n_{1}(U, V) n_{2}(U, V)$ são pares e $m_{1}(U, V) m_{2}(U, V)$ é ímpar.

Um polinômio em $K[U, V]$ chama-se par se todos os seus monômios são pares e chama-se ímpar se todos os seus monômios são ímpares.

Observamos que cada polinômio $P(U, V) \in \mathcal{P}$ que não seja par ou ímpar decompõe-se de maneira única como soma de um polinômio par e outro ímpar, isto é,

$$
P(U, V)=P_{0}(U, V)+P_{1}(U, V)
$$

onde $P_{0}(U, V) \in \mathcal{P}$ é par e $P_{1}(U, V) \in \mathcal{P}$ é ímpar.

Dado $p(U, V) \in \mathcal{P}$, diremos que $p_{e}$ é invariante quanto à escolha do 
idempotente $e$, ou simplesmente que é invariante, quando, para quaisquer $e, f \in I d A$, tem-se $p_{e}=p_{f}$. $\operatorname{Se} \operatorname{dim}\left(p_{e}\right)=\operatorname{dim}\left(p_{f}\right)$, para todo $e, f \in I d A$, diremos que $p_{e}$ tem dimensão invariante quanto à escolha do idempotente $e$, ou apenas que $p_{\boldsymbol{e}}$ tem dimensão invariante.

Lema 2.3 Em toda álgebra de Bernstein tem-se:

(a) $U_{e}^{2 k} \subseteq V_{e}$ e $U_{e}^{2 k+1} \subseteq U_{e}$ para $k \geq 1$;

(b) $V_{e}^{k} \subseteq L$ para $k \geq 2$;

(c) $U_{e}^{k} L=0$ para $k \geq 1$.

Demonstração. Provaremos as relações por indução em $k$. No item (a) observe que para $k=1, U_{e}^{2} \subseteq V_{e}$ e $U_{e}{ }^{3}=U_{e}{ }^{2} U_{e} \subseteq U_{e}$. Seja $k>1$. Por hipótese de indução as identidades valem para $k-1$. Então

$$
\begin{gathered}
U_{e}^{2 k}=\left(U_{e}^{2 k-2} U_{e}\right) U_{e} \subseteq\left(V_{e} U_{e}\right) U_{e} \subseteq V_{e}, \quad \mathrm{e} \\
U_{e}^{2 k+1}=\left(U_{e}^{2 k-1} U_{e}\right) U_{e} \subseteq U_{e}^{3} \subseteq U_{e} .
\end{gathered}
$$

Para (b) observe que $V_{e}^{2} \subseteq U_{e}$ e $U_{e} V_{e}^{2}=0$, de onde $V_{e}^{2} \subseteq L$. Então, como $L$ é ideal de $A$, segue da hipótese de indução que $V_{e}^{k}=V_{e}^{k-1} V_{e} \subseteq L V_{e} \subseteq L$.

No item (c), se $k$ é ímpar, segue do item (a) e da definição de $L$ que

$$
U_{e}^{k} L \subseteq U_{e} L=0
$$

Para $k$ par temos pelo item (a) e da identidade (1.1.15) que

$$
U_{e}^{k} L=\left(U_{e}^{k-1} U_{e}\right) L \subseteq U_{e}^{2} L \subseteq U_{e}\left(U_{e} L\right)=0
$$

O próximo corolário segue imediatamente dos itens (b) e (c) do lema acima. 
Corolário 2.4 Em toda álgebra de Bernstein tem-se:

(a) $V_{e}^{r} \subseteq U_{e}$ para $r \geq 2$;

(b) $U_{e}^{k} V_{e}^{r}=0$ para $r \geq 2$ e $k \geq 1$.

\subsection{Invariância de $P$-subespaços nas álgebras}

\section{de Bernstein}

Conforme discutido na seção anterior, os subespaços $\widetilde{U}=U_{e} \oplus U_{e}^{2} \mathrm{e}$ $\tilde{V}=\left(U_{e} V_{e}+U_{e}^{2} V_{e}\right) \oplus V_{e}$ não dependem da escolha do idempotente e assim, são invariantes. Temos ainda que $N=U_{e} \oplus V_{e}$, núcleo da função peso é invariante, pois $U_{e} \oplus V_{e}=U_{f} \oplus V_{f}$ para quaisquer idempotentes não nulos $e, f$ de $A$. Em [7], Etherington introduziu o ideal gerado pelos elementos da forma $x^{2}-\omega(x) x$, onde $x$ pertence a uma álgebra bárica $A$. Este ideal é conhecido como ideal de Etherington e será denotado por $P$. Se $A=K e \oplus U_{e} \oplus V_{e}$ é uma álgebra de Bernstein decomposta com relação a um idempotente $e$, então, conforme [23], o subespaço $\left(V_{e}^{2}+U_{e} V_{e}\right) \oplus V_{e}$ coincide com o ideal de Etherington de $A$. De fato, observe primeiro que

$$
\begin{gathered}
e\left(\left(V_{e}^{2}+U_{e} V_{e}\right) \oplus V_{e}\right)=e V_{e}^{2}+e U_{e} V_{e}+e V_{e}=V_{e}^{2}+U_{e} V_{e} \\
U_{e}\left(\left(V_{e}^{2}+U_{e} V_{e}\right) \oplus V_{e}\right)=U_{e}\left(U_{e} V_{e}\right) \oplus U_{e} V_{e} \subseteq U_{e} V_{e}+V_{e} \\
V_{e}\left(\left(V_{e}^{2}+U_{e} V_{e}\right) \oplus V_{e}\right)=V_{e} V_{e}^{2}+\left(U_{e} V_{e}\right) V_{e}+V_{e}^{2} \subseteq V_{e}^{2}+U_{e} V_{e}
\end{gathered}
$$


Assim, $\left(V_{e}^{2}+U_{e} V_{e}\right) \oplus V_{e}$ é um ideal de $A$. Agora, dado $x=\omega(x) e+u+v$ um elemento de $A$, com $u \in U_{e}$ e $v \in V_{e}$, temos que

$$
\begin{aligned}
x^{2}-\omega(x) x= & (\omega(x) e+u+v)^{2}-\omega(x)(\omega(x) e+u+v) \\
= & \omega(x)^{2} e+2 \omega(x) e u+u^{2}+2 u v+v^{2}-\omega(x)^{2} e \\
& -\omega(x) u-\omega(x) v \\
= & u^{2}-\omega(x) v+v^{2}+2 u v \in V_{e} \oplus\left(V_{e}^{2}+U_{e} V_{e}\right)
\end{aligned}
$$

donde $P \subseteq\left(V_{e}^{2}+U_{e} V_{e}\right) \oplus V_{e}$. Por outro lado, se $y \in U_{e} \oplus V_{e}=N$ então $y^{2}+\omega(y) y=y^{2} \in P$, ou seja, o quadrado de todo elemento do núcleo de $A$ pertence a $P$. Assim temos que $\left(U_{e} \oplus V_{e}\right)^{2}=U_{e}^{2}+U_{e} V_{e}+V_{e}^{2}$, donde $U_{e}^{2}, U_{e} V_{e}, V_{e}^{2} \subset P$. Por fim, fazendo $x=e+v, \operatorname{com} v \in V_{e}, \operatorname{temos} \omega(x)=1 \mathrm{e}$

$$
\begin{aligned}
x^{2}-x & =(e+v)^{2}-(e+v) \\
& =e^{2}+2 e v+v^{2}-e-v \\
& =v^{2}-v .
\end{aligned}
$$

Como $v^{2} \in P$, segue que $v \in P$ e assim, $\left(V_{e}^{2}+U_{e} V_{e}\right) \oplus V_{e} \subseteq P$. Das inclusões temos que $\left(V_{e}^{2}+U_{e} V_{e}\right) \oplus V_{e}=P$, como queríamos.

Da última igualdade segue que $\left(V_{e}^{2}+U_{e} V_{e}\right) \oplus V_{e}$ é invariante nas álgebras de Bernstein.

Segundo demonstrado em [30], a soma e o produto de $P$-subespaços invariantes produz novos subespaços invariantes. Daremos a seguir alguns 
exemplos de $P$-subespaços invariantes:

$$
\begin{aligned}
N P & =\left(U_{e} V_{e}+V_{e}^{2}\right) \oplus U_{e}\left(U_{e} V_{e}\right) \\
N^{3} & =\left(U_{e}^{3}+U_{e}^{2} V_{e}+\left(U_{e} V_{e}\right) V_{e}+V_{e}^{3}\right) \oplus U_{e}\left(U_{e} V_{e}\right) \\
{\widetilde{U_{e}}}^{2}+P^{2} & =\left(U_{e}^{3}+\left(U_{e} V_{e}\right) V_{e}+V_{e}^{2}+V_{e}^{3}\right) \oplus U_{e}^{2} \\
N \widetilde{U}^{2} & =\left(U_{e}^{3}+U_{e}^{2} V_{e}+U_{e} V_{e}\right) \oplus U_{e}^{2}
\end{aligned}
$$

É claro que um $P$-subespaço invariante tem dimensão invariante. Provaremos a seguir a invariância da dimensão de alguns $P$-supespaços.

Lema 2.5 Seja $A=K e \oplus U_{e} \oplus V_{e}$ uma álgebra de Bernstein. Então $U_{e}^{2}$, $U_{e}\left(U_{e} V_{e}\right)$ e $U_{e} V_{e}+V_{e}^{2}$ têm dimensão invariante.

Demonstração. Conforme vimos anteriormente, $\widetilde{U}=U_{e} \oplus U_{e}{ }^{2}$ é invariante. Logo, $\operatorname{dim} U_{e}^{2}=\operatorname{dim} \widetilde{U}-\operatorname{dim} U_{e}$ e portanto $U_{e}^{2}$ tem dimensão invariante.

Observando que $P$, o ideal de Etherington, e $V_{e}$, pelo Lema 1.7, têm dimensão invariante, segue que $U_{e} V_{e}+V_{e}^{2} \subseteq U_{e}$ tem dimensão invariante.

Por fim, desde que $U_{e}\left(U_{e} V_{e}\right) \subseteq V_{e}$, usando (2.3.1) e o resultado acima temos que $U_{e}\left(U_{e} V_{e}\right)$ também tem dimensão invariante.

Lema 2.6 Seja $A=K e \oplus U_{e} \oplus V_{e}$ uma álgebra de Bernstein tal que $U_{e}^{2} V_{e}=$ 0. Se $m(U, V)=\alpha(U, V) \beta(U, V) \in \mathcal{P}$ é um monômio de grau $k \geq 3$, com $\alpha(U, V)$ e $\beta(U, V)$ impares, então $m_{e}=0$.

Demonstração. Seja $m_{e}=\alpha_{e} \beta_{e}$, onde $\alpha(U, V)$ e $\beta(U, V)$ são ímpares, com $\partial \alpha(U, V) \geq 2$. Então, existem $\mu(U, V), \delta(U, V) \in \mathcal{P}$ pares tais que $\alpha_{e}=\mu_{e} \delta_{e}$. Assim, temos que

$$
m_{e}=\alpha_{e} \beta_{e}=\left(\mu_{e} \delta_{e}\right) \beta_{e} \subseteq U_{e}^{2} V_{e}=0
$$


Seja $A=K e \oplus U_{e} \oplus V_{e}$ uma álgebra de Bernstein. Para cada $\mathrm{u} \in U_{e}$ a função $\psi_{\mathbf{u}}: U_{e} \rightarrow U_{e}$, definida por

$$
\psi_{\mathbf{u}}(u)=u-2 \mathbf{u}^{2} u
$$

é um isomorfismo de espaços vetoriais. De fato, se $\psi_{\mathbf{u}}(u)=0, u=2 \mathbf{u}^{2} u$ e como $\mathbf{u}^{2} u+2 \mathbf{u}(\mathbf{u} u)=0$, temos $u=4 \mathbf{u}(\mathbf{u} u)$. Por outro lado, de $u=2 \mathbf{u}^{2} u$ segue que $\mathbf{u} u=2 \mathbf{u}\left(\mathbf{u}^{2} u\right)=-2 u \mathbf{u}^{3}=0$. Portanto, $u=0$ e a afirmação está demonstrada.

A partir deste momento restringiremos nosso estudo às álgebras chamadas de Bernstein-Picanço em referência aos trabalhos de J.Picanço (ver [6] e [30]). Uma álgebra de Bernstein $A$ chama-se Bernstein-Picanço se possui um idempotente $e$ tal que $U_{e}^{2} V_{e}=0$ e também $(u v) v=0$, para todo $u \in U_{e}$ e $v \in V_{e}$. Observamos que esta subclasse das álgebras de Bernstein contém as álgebras de Bernstein-Jordan.

Sejam $A=K e \oplus U_{e} \oplus V_{e}$ uma álgebra de Bernstein e u um elemento fixado de $U_{e}$. A proposição seguinte mostra como as funções lineares $\psi_{\mathbf{u}} \mathrm{e}$ $\tau_{\mathbf{u}}=\varphi_{\mathbf{u}} \oplus \sigma_{\mathbf{u}}$ dadas por



$$
\begin{aligned}
& v \quad \mapsto \quad v-2\left(\mathbf{u}+\mathbf{u}^{2}\right) v
\end{aligned}
$$

onde $f=e+\mathbf{u}+\mathbf{u}^{2}, u \in U_{e}$ e $v \in V_{e}$, se relacionam nas álgebras de Bernstein-Picanço. 
Proposição 2.7 Seja $A=K e \oplus U_{e} \oplus V_{e}$ uma álgebra de Bernstein-Picanço. Então, para cada $u, u_{1}, u_{2} \in U_{e}$ e $v, v_{1}, v_{2} \in V_{e}$, temos:
(a) $\tau_{\mathbf{u}}\left(u_{1}\right) \tau_{\mathbf{u}}\left(u_{2}\right)=\tau_{\mathbf{u}}\left(u_{1} u_{2}\right)$;
(b) $\tau_{\mathbf{u}}(u) \tau_{\mathbf{u}}(v)=\tau_{\mathbf{u}}\left(\psi_{\mathbf{u}}(u) v\right)$;
(c) $\tau_{\mathbf{u}}\left(v_{1}\right) \tau_{\mathbf{u}}\left(v_{2}\right)=\tau_{\mathbf{u}}\left(v_{1} v_{2}\right)=v_{1} v_{2}$;
(d) $\psi_{\mathbf{u}}\left(\psi_{\mathbf{u}}(u) v\right)=u v$.

Demonstração. Para o item (a) temos, a partir das identidades (1.1.9) e (1.1.13), que

$$
\begin{aligned}
\tau_{\mathbf{u}}\left(u_{1}\right) \tau_{\mathbf{u}}\left(u_{2}\right) & =\left(u_{1}+2 \mathbf{u} u_{1}\right)\left(u_{2}+2 \mathbf{u} u_{2}\right) \\
& =u_{1} u_{2}+2 u_{1}\left(\mathbf{u} u_{2}\right)+2 u_{2}\left(\mathbf{u} u_{1}\right)+4\left(\mathbf{u} u_{1}\right)\left(\mathbf{u} u_{2}\right) \\
& =u_{1} u_{2}-2 \mathbf{u}\left(u_{1} u_{2}\right)-2 \mathbf{u}^{2}\left(u_{1} u_{2}\right) \\
& =\tau_{\mathbf{u}}\left(u_{1} u_{2}\right) .
\end{aligned}
$$

No item (b), das identidades (1.1.16) a (1.1.18) e de $U_{e}^{2} V_{e}=0$ temos

$$
\begin{aligned}
\tau_{\mathbf{u}}(u) \tau_{\mathbf{u}}(v) & =(u+2 \mathbf{u} u)(v-2 \mathbf{u} v) \\
& =u v-2 u(\mathbf{u} v)+2(\mathbf{u} u) v-4(\mathbf{u} u)(\mathbf{u} v) \\
& =u v+2 \mathbf{u}(u v)+2 \mathbf{u}^{2}(u v) .
\end{aligned}
$$

E, por outro lado,

$$
\begin{aligned}
\tau_{\mathbf{u}}\left(\psi_{\mathbf{u}}(u) v\right) & =\tau_{\mathbf{u}}\left(u v-2\left(\mathbf{u}^{2} u\right) v\right) \\
& =u v-2\left(\mathbf{u}^{2} u\right) v+2 \mathbf{u}(u v)-4 \mathbf{u}\left(\left(\mathbf{u}^{2} u\right) v\right) \\
& =u v+2 \mathbf{u}(u v)+2 \mathbf{u}^{2}(u v) .
\end{aligned}
$$

Para o item (c) observe que da Proposição 1.10 e da identidade 
(1.1.18) temos

$$
\begin{aligned}
\tau_{\mathbf{u}}\left(v_{1}\right) \tau_{\mathbf{u}}\left(v_{2}\right) & =\left(v_{1}-2 \mathbf{u} v_{1}\right)\left(v_{2}-2 \mathbf{u} v_{2}\right) \\
& =v_{1} v_{2}-2\left(\mathbf{u} v_{1}\right) v_{2}-2\left(\mathbf{u} v_{2}\right) v_{1}+4\left(\mathbf{u} v_{1}\right)\left(\mathbf{u} v_{2}\right) \\
& =v_{1} v_{2} .
\end{aligned}
$$

$\mathrm{E}, \tau_{\mathbf{u}}\left(v_{1} v_{2}\right)=v_{1} v_{2}+2 \mathbf{u}\left(v_{1} v_{2}\right)=v_{1} v_{2}$.

Por fim, das identidades (1.1.16) a (1.1.18) e (1.2.7) segue que

$$
\begin{aligned}
\psi_{\mathbf{u}}\left(\psi_{\mathbf{u}}(u) v\right) & =\psi_{\mathbf{u}}\left(u v-2\left(\mathbf{u}^{2} u\right) v\right) \\
& =u v-2\left(\mathbf{u}^{2} u\right) v-2 \mathbf{u}^{2}(u v)+4 \mathbf{u}^{2}\left(\left(\mathbf{u}^{2} u\right) v\right) \\
& =u v
\end{aligned}
$$

O que verifica o item (d).

Corolário 2.8 Seja $A=K e \oplus U_{e} \oplus V_{e}$ uma álgebra de Bernstein-Picanço. Então, para cada idempotente $f$ de A temos $U_{f}{ }^{2} V_{f}=0$ e $(u v) v=0$ para todo $u \in U_{f}$ e $v \in V_{f}$.

Demonstração. Sejam $A=K e \oplus U_{e} \oplus V_{e}$ uma álgebra de BernsteinPicanço e $f=e+\mathbf{u}+\mathbf{u}^{2} \in I d A$, para $\mathbf{u} \in U_{e}$, um idempotente não nulo. Temos que $U_{f}^{2} V_{f}=\left\langle\left(\tau_{\mathbf{u}}\left(u_{1}\right) \tau_{\mathbf{u}}\left(u_{2}\right)\right) \tau_{\mathbf{u}}(v) \quad\right| u_{1}, u_{2} \in U_{e}$ e $\left.v \in V_{e}\right\rangle$. Mas, $\left(\tau_{\mathbf{u}}\left(u_{1}\right) \tau_{\mathbf{u}}\left(u_{2}\right)\right) \tau_{\mathbf{u}}(v)=\tau_{\mathbf{u}}\left(u_{1} u_{2}\right) \tau_{\mathbf{u}}(v)=\tau_{\mathbf{u}}\left(\left(u_{1} u_{2}\right) v\right)=\left(u_{1} u_{2}\right) v=0$. Além disso, $\left(\tau_{\mathbf{u}}(u) \tau_{\mathbf{u}}(v)\right) \tau_{\mathbf{u}}(v)=\tau_{\mathbf{u}}\left(\psi_{\mathbf{u}}(u) v\right) \tau_{\mathbf{u}}(v)=\tau_{\mathbf{u}}\left(\psi_{\mathbf{u}}\left(\psi_{\mathbf{u}}(u) v\right) v\right)=$ $\tau_{\mathbf{u}}((u v) v)=0$. O que completa a demonstração.

Corolário 2.9 Seja A uma álgebra de Bernstein-Picanço. Se $X, X_{1}, X_{2} \subseteq$ $U_{e}$ e $W, W_{1}, W_{2} \subseteq V_{e}$ são subespaços de $A$, então: 
(a) $\left(X W_{1}\right) W_{2}=\left(X W_{2}\right) W_{1}$;

(b) $\tau_{\mathbf{u}}\left(X_{1}\right) \tau_{\mathbf{u}}\left(X_{2}\right)=\tau_{\mathbf{u}}\left(X_{1} X_{2}\right)$;

(c) $\tau_{\mathbf{u}}(X) \tau_{\mathbf{u}}(W)=\tau_{\mathbf{u}}\left(\psi_{\mathbf{u}}(X) W\right)$;

(d) $\tau_{\mathbf{u}}\left(W_{1}\right) \tau_{\mathbf{u}}\left(W_{2}\right)=\tau_{\mathbf{u}}\left(W_{1} W_{2}\right)=W_{1} W_{2}$.

Segue da Proposição 1.10 e do fato de $U_{e}^{2} V_{e} \subseteq V_{e}^{2}$ que toda álgebra de Bernstein-Jordan é uma álgebra de Bernstein-Picanço, como havíamos destacado. Entretanto, conforme [30], a recíproca deste resultado não é verdadeira, visto que, para a álgebra de Bernstein $A=K e \oplus\langle u\rangle \oplus\langle v\rangle$ com tábua de multiplicação dada por

$$
e^{2}=e, \quad e u=\frac{1}{2} u, \quad v^{2}=u
$$

e os demais produtos zero, safisfaz $U_{e}^{2} V_{e}=0$ e $(u v) v=0$, pois $u^{2}=0$ e $u v=0$, mas $v^{2} \neq 0$ e assim $A$ não é Bernstein-Jordan.

Lema 2.10 Sejam A uma álgebra de Bernstein e $p(U, V) \in \mathcal{P}$. Então:

(a) Se A é uma álgebra de Jordan, $V_{e} p_{e} \subseteq p_{e}$;

(b) Se A é uma álgebra de Bernstein-Picanço, $U_{e}^{2} p_{e} \subseteq p_{e}$.

Demostração Para o item (a) é suficiente mostrar a relação para cada monômio $m(U, V) \in \mathcal{P}$. Provaremos a relação por indução sobre o grau $k$ de $m(U, V)$. Para $k=1$, se $m(U, V)$ é ímpar, temos $V_{e} m_{e}=V_{e}^{2}=0$. Por outro lado, se $m(U, V)$ é par, segue que $V_{e} m_{e}=V_{e} U_{e} \subseteq U_{e}$. Seja $k>1$ e assuma, por hipótese de indução, que $V_{e} p_{e} \subseteq p_{e}$ para cada monômio $p(U, V)$ com grau menor que $k$ e seja $m(U, V) \in \mathcal{P}$ monômio com $\partial m(U, V)=k$. Então existem monômios $\alpha(U, V), \beta(U, V) \in \mathcal{P}$ de graus menores que $k$ tais 
que $m(U, V)=\alpha(U, V) \beta(U, V)$. Do Corolário 2.9 e da hipótese de indução segue que

$$
\begin{aligned}
V_{e} m_{e} & =V_{e}\left(\alpha_{e} \beta_{e}\right)=\left(V_{e} \alpha_{e}\right) \beta_{e}+\alpha_{e}\left(V_{e} \beta_{e}\right) \\
& \subseteq \alpha_{e} \beta_{e}=m_{e} .
\end{aligned}
$$

Para o item (b), de maneira análoga, é suficiente mostrar a relação para cada monômio $m(U, V) \in \mathcal{P}$. Provaremos por indução sobre o grau $k$ de $m(U, V)$. Para $k=1$ temos que $m_{e}=U_{e}$ ou $m_{e}=V_{e}$, assim $U_{e}{ }^{2} m_{e}=U_{e}{ }^{3} \subset$ $U_{e}$ e no segundo caso $U_{e}^{2} V_{e}=0$. Assuma então que o lema é verdadeiro para todo monômio de grau menor que $k$ e seja $m(U, V)$ um monômio de grau $k>1$. Então, pelo Lema 2.6, temos três possibilidades. No primeiro caso, se $m(U, V)$ é ímpar, então $U_{e}^{2} m_{e} \subset U_{e}^{2} V_{e}=0$ e, no segundo, se $m_{e} \subset V_{e}^{2}, U_{e}^{2} m_{e}=U_{e}^{2} V_{e}^{2}=0$, pelo Corolário 2.4. Por fim, se $m(U, V)=$ $\alpha(U, V) \beta(U, V) \in \mathcal{P}$, com $\alpha(U, V), \beta(U, V)$ monômios de grau menor que $k$, com $\alpha(U, V)$ par e $\beta(U, V)$ ímpar temos, pelo Corolário 2.9(a) e da hipótese de indução que

$$
U_{e}^{2} m_{e}=U_{e}^{2}\left(\alpha_{e} \beta_{e}\right)=\beta_{e}\left(\alpha_{e} U_{e}^{2}\right) \subset \alpha_{e} \beta_{e}=m_{e}
$$

o que completa a demonstração.

Lema 2.11 Sejam A uma álgebra de Bernstein-Picanço e $p(U, V) \in \mathcal{P}$ par. Então $\psi_{\mathbf{u}}\left(p_{e}\right)=p_{e}$.

Demonstração. Como $\psi_{\mathbf{u}}$ é isomorfismo, basta verificar que $\psi_{\mathbf{u}}\left(p_{e}\right) \subseteq p_{e}$. Seja $t \in p_{e}$. Desde que $\mathbf{u}^{2} \in U_{e}^{2}$, temos, pelo Lema 2.10, que $\psi_{\mathbf{u}}(t)=$ $t-2 \mathbf{u}^{2} t \in p_{e}+U_{e}^{2} p_{e}=p_{e}$. 
Teorema 2.12 Se A é uma álgebra de Bernstein-Picanço e $m(U, V)$, $n(U, V) \in \mathcal{P}$ são monômios tais que $m(U, V)$ é par e $n(U, V)$ é ímpar, então

$$
m_{f}=\tau_{\mathbf{u}}\left(m_{e}\right) \quad e \quad n_{f}=\tau_{\mathbf{u}}\left(n_{e}\right)
$$

Demonstração. Sejam $K e \oplus U_{e} \oplus V_{e}=K f \oplus U_{f} \oplus V_{f}$ duas decomposições de Peirce da álgebra $A$, onde $f=e+\mathbf{u}+\mathbf{u}^{2}$, com $\mathbf{u} \in U_{e}$. Provaremos a primeira relação do teorema por indução sobre o grau $k$ de $m(U, V)$. Se $k=1$, então $m(U, V)=U$ e $m_{f}=\tau_{\mathbf{u}}\left(m_{e}\right)$, pois $\tau_{\mathbf{u}}$ é isomorfismo de espaços vetoriais. Seja $k>1$ e assuma, por hipótese de indução, que (2.3.5) vale para todo monômio de grau menor que $k$. Se $k=2$ e $m(U, V)=V^{2}$ segue do Corolário 2.9 que $m_{f}=V_{f}^{2}=\tau_{\mathbf{u}}\left(V_{e}^{2}\right)=\tau_{\mathbf{u}}\left(m_{e}\right)$. Em outro caso, existem monômios $\mu(U, V), \rho(U, V) \in \mathcal{P}$, de grau menor que $k$, com $\mu(U, V)$ par e $\rho(U, V)$ ímpar, tais que $m_{e}=\mu_{e} \rho_{e}$. Então, do Corolário 2.9 e do Lema 2.11 temos que $m_{f}=\mu_{f} \rho_{f}=\tau_{\mathbf{u}}\left(\mu_{e}\right) \tau_{\mathbf{u}}\left(\rho_{e}\right)=\tau_{\mathbf{u}}\left(\psi_{\mathbf{u}}\left(\mu_{e}\right) \rho_{e}\right)=\tau_{\mathbf{u}}\left(\mu_{e} \rho_{e}\right)=\tau_{\mathbf{u}}\left(m_{e}\right)$.

Agora seja $n(U, V) \in \mathcal{P}$. Analogamente, provaremos a segunda igualdade do teorema por indução sobre o grau $k$ de $n(U, V)$. Se $k=1$, então $n(U, V)=V$ e $n_{f}=\tau_{\mathbf{u}}\left(n_{e}\right)$. Seja $k>1$ e novamente assuma que a segunda identidade de (2.3.5) vale para todo monômio de grau menor que $k$. Então existem monômios $\gamma(U, V), \delta(U, V) \in \mathcal{P}$ pares, de grau menor que $k$, tais que $n_{e}=\gamma_{e} \delta_{e}$. Assim, do Corolário 2.9 temos que $n_{f}=\gamma_{f} \delta_{f}=\tau_{\mathbf{u}}\left(\gamma_{e}\right) \tau_{\mathbf{u}}\left(\delta_{e}\right)=$ $\tau_{\mathbf{u}}\left(\gamma_{e} \delta_{e}\right)=\tau_{\mathbf{u}}\left(n_{e}\right)$.

Corolário 2.13 Em uma álgebra de Bernstein-Picanço todo P-subespaço tem dimensão invariante. 
Demonstração. Sejam $A=K e \oplus U_{e} \oplus V_{e}$ uma álgebra de Bernstein-Picanço e $p(U, V) \in \mathcal{P}$. Então existem monômios $m_{i}(U, V) \in \mathcal{P}$ tais que $p_{e}=\sum_{i=1}^{r} m_{i, e}$. Desta forma, $p_{f}=\sum_{i=1}^{r} m_{i, f}=\sum_{i=1}^{r} \tau_{\mathbf{u}}\left(m_{i, e}\right)=\tau_{\mathbf{u}}\left(\sum_{i=1}^{r} m_{i, e}\right)=\tau_{\mathbf{u}}\left(p_{e}\right)$. Então $p_{e}$ tem dimensão invariante.

O próximo resultado segue imediatamente do corolário acima.

Corolário 2.14 Todo P-subespaço de uma álgebra de Bernstein-Jordan tem dimensão invariante.

Corolário 2.15 Todo P-subespaço de uma álgebra de Bernstein, da forma $h_{e}, \operatorname{com} h(U, V) \in \mathcal{P}$ impar, tem dimensão invariante.

Demonstração. Seja $A=K e \oplus U_{e} \oplus V_{e}$ uma álgebra de Bernstein e considere $\bar{A}=K \bar{e} \oplus \bar{U}_{\bar{e}} \oplus \bar{V}_{\bar{e}}$ a decomposição da álgebra quociente $A / L$, associada ao idempotente $\bar{e}=e+L$. Então, para todo $h(U, V) \in \mathcal{P}$ ímpar, temos $\bar{h}_{\bar{e}}=\left(h_{e} \oplus L\right) / L \simeq h_{e} /\left(h_{e} \cap L\right) \simeq h_{e}$, desde que $L \subseteq U_{e} . \mathrm{E} \operatorname{assim}, \operatorname{dim}\left(\bar{h}_{\bar{e}}\right)=$ $\operatorname{dim}\left(h_{e}\right)$.

Como conseqüência do Corolário acima, temos o seguinte critério para determinar quando um $P$-subespaço de uma álgebra de Bernstein tem dimensão invariante.

Corolário 2.16 Seja $p(U, V)=g(U, V) \oplus h(U, V) \in \mathcal{P}$ com $g(U, V)$ par e $h(U, V)$ ímpar. Então $p_{e}$ tem dimensão invariante se, e somente se, $g_{e}$ tem dimensão invariante.

Este último Corolário justifica o método usado no Lema 2.5 para a obtenção de $P$-subespaços de dimensão invariante nas álgebras de Bernstein. 
O próximo teorema traz um importante critério para determinar se um $P$-subespaço de uma álgebra de Bernstein-Picanço é invariante.

Teorema 2.17 Sejam $A=K e \oplus U_{e} \oplus V_{e}$ uma álgebra de Bernstein-Picanço e $p(U, V) \in \mathcal{P}$. Então $p_{e}$ é invariante se, e somente se, $U_{e} p_{e} \subseteq p_{e}$.

Demonstração. Sejam $A=K e \oplus U_{e} \oplus V_{e}$ uma álgebra de Bernstein-Picanço e $p(U, V)=g(U, V) \oplus h(U, V) \in \mathcal{P}$ com $g(U, V)$ par e $h(U, V)$ ímpar. Para cada $\mathbf{u} \in U_{e}$ seja $f=e+\mathbf{u}+\mathbf{u}^{2} \in I d A$. Do Teorema 2.12 temos que

$$
\begin{aligned}
p_{f} & =g_{f} \oplus h_{f} \\
& =\tau_{\mathbf{u}}\left(g_{e}\right) \oplus \tau_{\mathbf{u}}\left(h_{e}\right) \\
& =\left\{\tau_{\mathbf{u}}(x) \mid x \in g_{e}\right\} \oplus\left\{\tau_{\mathbf{u}}(w) \mid w \in h_{e}\right\} \\
& =\left\{x+2 \mathbf{u} x+w-2 \mathbf{u} w \mid x \in g_{e}, w \in h_{e}\right\} \\
& \subseteq p_{e}+U_{e} p_{e} .
\end{aligned}
$$

Suponhamos primeiro que $p_{e}$ é invariante. Assim para cada $\mathbf{u} \in U_{e}$ temos $f=e+\mathbf{u}+\mathbf{u}^{2} \in I d A$ e $p_{f}=p_{e}$. Por (2.3.5), para cada $z=x+w \in p_{e}$ $\operatorname{com} x \in g_{f}$ e $w \in h_{f}, \tau_{\mathbf{u}}(z)=x+w+2 \mathbf{u}(x-w) \in p_{f}$. Portanto existe $\bar{z}=\bar{x}+\bar{w} \in p_{e} \operatorname{com} \bar{x} \in g_{f}$ e $\bar{w} \in h_{f}$ tal que $\tau_{\mathbf{u}}(z)=\bar{z}$, isto é

$$
\bar{x}+\bar{w}=(x-2 \mathbf{u} w)+(w+2 \mathbf{u} x)
$$

$\log 0$

$$
2 \mathbf{u} w=x-\bar{x} \in p_{e} \quad \text { e } \quad 2 \mathbf{u} x=\bar{w}-w \in p_{e} .
$$

Assim, $\mathbf{u} z=\mathbf{u}(x+w) \in p_{e}$. Já que esta relação é válida para cada $\mathbf{u} \in U_{e}$ e $z \in p_{e}$ temos que $U_{e} p_{e} \subset p_{e}$ como queríamos provar. 
Admitimos agora que $U p_{e} \subseteq p_{e}$. A identidade (2.3.5) pode ser escrita como

$$
p_{f}=\left\{x+w+2 \mathbf{u}(x-w) \mid x \in g_{e}, w \in h_{e}\right\}
$$

de onde vem que $p_{f} \subseteq p_{e}+U_{e} p_{e}$. Usando a hipótese temos que $p_{f} \subseteq p_{e}$ e, como pelo Corolário 2.13 ambos subespaços tem a mesma dimensão, temos que $p_{f}=p_{e}$, e $p_{e}$ é invariante.

Corolário 2.18 Sejam $A=K e \oplus U_{e} \oplus V_{e}$ uma álgebra de Bernstein-Jordan e $p(U, V) \in \mathcal{P}$. São equivalentes:
(a) $p_{e}$ é invariante;
(b) $U_{e} p_{e} \subseteq p_{e}$;
(c) $p_{e}$ é um ideal de $A$.

Demonstração. Segue do Teorema 2.17 que (a) é equivalente a (b).

Vamos mostrar que (b) é equivalente a (c). Desde que $A=K e \oplus U_{e} \oplus V_{e}$, temos que $p_{e}$ é um ideal de $A$ se, e somente se, $e p_{e} \subseteq p_{e}, U_{e} p_{e} \subseteq p_{e}$ e $V_{e} p_{e} \subseteq p_{e}$. Como $p(U, V)=g(U, V) \oplus h(U, V) \in \mathcal{P}$ com $g(U, V)$ par e $h(U, V)$ ímpar temos, da definição de $U_{e}$ e do Lema 2.10, respectivamente que $e p_{e} \subseteq p_{e}$ e $V_{e} p_{e} \subseteq p_{e}$. Assim, $p_{e}$ é um ideal de $A$ se, e somente se $U_{e} p_{e} \subseteq p_{e}$, o que completa a demonstração. 
CAPÍTULO 3

\section{Álgebras de Bernstein e}

\section{álgebras genéticas}

Neste capítulo estudaremos as relações entre as estruturas algébricas que têm sua motivação na genética e as álgebras de Bernstein.

\section{1 Álgebras báricas: uma classificação}

No que segue, assumimos que $A$ é uma álgebra comutativa de dimensão $n$, não necessariamente associativa sobre um corpo $K$, com um homomorfismo não nulo $\omega: A \rightarrow K$ chamado homomorfismo de peso. Denotaremos por $K\left[\lambda_{1}, \cdots, \lambda_{n}\right]$ o anel de polinômios sobre $K$ nas indeterminadas $\lambda_{1}, \cdots, \lambda_{n}$ e por $|K|$ o número de elementos do corpo $K$. Como já foi destacado, o par $(A, \omega)$ é chamado álgebra bárica. O núcleo do homomorfismo $\omega$ é um ideal de codimensão 1 chamado barideal.

Lema 3.1 Sejam $K$ um corpo com ao menos $k$ elementos e $p\left(\lambda_{1}, \cdots, \lambda_{m}\right) \in$ $K\left[\lambda_{1}, \cdots, \lambda_{m}\right]$ um polinômio não nulo com grau em $\lambda_{i}<k-1$ para cada 
$i=1, \cdots, m$. Então existem $\alpha_{1}, \cdots, \alpha_{m} \in K$ tais que

$$
\sum_{i=1}^{m} \alpha_{i} \neq 0, \quad p\left(\alpha_{1}, \cdots, \alpha_{m}\right) \neq 0
$$

Demonstração. Faremos uma indução sobre $m$. Seja $m=1$ e $p(\lambda) \in K[\lambda]$ um polinômio não nulo na variável $\lambda$ de grau $t<k-1$. Já que um polinômio não nulo de grau $t$ possui no máximo $t$ raízes em $K$ e $|K|>t+1$ existe obviamente $\alpha \in K, \alpha \neq 0$ tal que $p(\alpha) \neq 0$. Seja $m>1$. Assumimos o lema para polinômios em $s$ variáveis com $1 \leq s<m$. Seja $p\left(\lambda_{1}, \cdots, \lambda_{m}\right) \in$ $K\left[\lambda_{1}, \cdots, \lambda_{m}\right]$ um polinômio não nulo com grau de $p\left(\lambda_{1}, \cdots, \lambda_{m}\right)$ em $\lambda_{i}<$ $k-1$ para cada $i=1, \cdots, m$. Então $p\left(\lambda_{1}, \cdots, \lambda_{m}\right)$ pode ser representado de maneira única

$$
p\left(\lambda_{1}, \cdots, \lambda_{m}\right)=\sum_{i=1}^{t} a_{i}\left(\lambda_{2}, \cdots, \lambda_{m}\right) \lambda_{1}^{i}
$$

$\operatorname{com} a_{i}\left(\lambda_{2}, \cdots, \lambda_{m}\right) \in K\left[\lambda_{2}, \cdots, \lambda_{m}\right]$ e $a_{t}\left(\lambda_{2}, \cdots, \lambda_{m}\right)$ não nulo. Por hipótese de indução, existem $\alpha_{2}, \cdots, \alpha_{m} \in K$ tais que $a_{t}\left(\alpha_{2}, \cdots, \alpha_{m}\right) \neq 0$.

Sejam $\beta_{i}=a_{i}\left(\alpha_{2}, \cdots, \alpha_{m}\right), \quad i=1, \cdots, t, \mathrm{e}$

$$
q\left(\lambda_{1}\right):=p\left(\lambda_{1}, \alpha_{2}, \cdots, \alpha_{m}\right)=\beta_{t} \lambda_{1}^{t}+\cdots+\beta_{2} \lambda_{1}^{2}+\beta_{1} \lambda_{1}+\beta_{0}
$$

um polinômio em $K\left[\lambda_{1}\right]$ de grau $t<k-1$. Já que $q\left(\lambda_{1}\right)$ possui no máximo $t$ raízes em $K$, e $|K|>t+1$, existe $\alpha_{1} \in K$ tal que $\alpha_{1} \neq-\left(\alpha_{2}+\cdots+\alpha_{m}\right)$ e $q\left(\alpha_{1}\right) \neq 0$. Assim,

$$
p\left(\alpha_{1}, \cdots, \alpha_{m}\right)=q\left(\alpha_{1}\right) \neq 0 \quad \text { e } \quad \alpha_{1}+\alpha_{2}+\cdots+\alpha_{m} \neq 0 .
$$

Portanto, o lema está provado. 
Lema 3.2 Seja A uma álgebra bárica sobre um corpo $K$, com $|K|>r+2$, e $p(\lambda)=\lambda^{r+1}+\alpha_{1} \lambda^{r}+\cdots+\alpha_{r} \lambda$ um polinômio em $K[\lambda]$ tal que $p(1)=0$. Então $p(x)=0$ para todo $x \in A$ de peso 1 se, e somente se, o polinômio train

$$
\bar{p}(x):=x^{r+1}+\alpha_{1} \omega(x) x^{r}+\cdots+\alpha_{r-1} \omega(x)^{r-1} x^{2}+\alpha_{r} \omega(x)^{r} x,
$$

é uma identidade para cada elemento $x \in A$.

Demonstração. Assumimos que $p(x)=0$ para todo $x \in A$ de peso 1 . Então $\bar{p}(x)=p(x)=0$ para cada $x \in A$ de peso 1 . Se $x \in A \operatorname{com} \omega(x) \neq 0$, então $\omega\left(\frac{x}{\omega(x)}\right)=1$ e assim,

$$
\begin{aligned}
0 & =p\left(\frac{x}{\omega(x)}\right) \\
& =\left(\frac{x}{\omega(x)}\right)^{r+1}+\alpha_{1}\left(\frac{x}{\omega(x)}\right)^{r}+\cdots+\alpha_{r}\left(\frac{x}{\omega(x)}\right) \\
& =\frac{1}{\omega(x)^{r+1}}\left[x^{r+1}+\alpha_{1} \omega(x) x^{r}+\cdots+\alpha_{r} \omega(x)^{r} x\right] \\
& =\frac{1}{\omega(x)^{r+1}} \bar{p}(x),
\end{aligned}
$$

donde segue que $\bar{p}(x)=0$ para todo $x \in A \operatorname{com} \omega(x) \neq 0$.

Agora, seja $\mathbb{B}=\left\{a_{1}, \cdots, a_{n}\right\}$ uma base para $A$ tal que cada $a_{i}$ tenha peso 1. Para cada $x \in A$, denotaremos por $\pi_{i}(x)$ a coordenda $i$-ésima de $x$ com respeito a base $\mathbb{B}$, isto é, $x=\sum_{i=1}^{n} \pi_{i}(x) a_{i}$. A forma polinômial $f_{i}: K^{n} \rightarrow K$ definida por $f_{i}\left(\mu_{1}, \cdots, \mu_{n}\right):=\pi_{i}\left(\bar{p}\left(\sum_{k=1}^{n} \mu_{k} a_{k}\right)\right)$ é igual a zero para todo $\bar{\mu}:=$ $\left(\mu_{1}, \cdots, \mu_{n}\right) \in K^{n} \operatorname{com} s(\bar{\mu}):=\sum_{k=1}^{n} \mu_{k} \neq 0$. Então, pelo Lema $3.1 f_{i} \equiv 0$. Portanto, $\bar{p}(x)=\sum_{i=1}^{n} f_{i}\left(\pi_{1}(x), \cdots, \pi_{n}(x)\right) a_{i}=0$ para todo $x \in A$. 
Para o decorrer desse capítulo assumimos que $K$ é um corpo com um número de elementos para os quais os lemas acima são verificados.

Em muitas álgebras que surgem em conexão com problemas genéticos, para cada $x \in A$, os coeficientes do polinômio característico da transfomação linear $R_{x}$ dependem somente do valor do homomorfismo $\omega$ em $x$. Álgebras báricas satisfazendo esta propriedade chamam-se álgebras train. Assim, uma álgebra bárica $A$ é train, se existem constantes $\beta_{1}, \ldots, \beta_{n} \in K$, tais que

$$
\operatorname{det}\left(\lambda I-R_{x}\right)=\lambda^{n}-\beta_{1} \omega(x) \lambda^{n-1}+\ldots+(-1)^{n} \beta_{n} \omega(x)^{n},
$$

para todo $x \in A$. Equivalentemente, o polinômio característico de $R_{x}$ é

$$
\operatorname{det}\left(\lambda I-R_{x}\right)=\lambda^{n}-\beta_{1} \lambda^{n-1}+\ldots+(-1)^{n} \beta_{n},
$$

para cada $x$ em $A$ de peso 1 . As raízes do polinômio característico são chamadas raízes train da álgebra. Notemos que 1 é uma raiz train já que para cada elemento $x \in A \operatorname{com} \omega(x)=1$ temos que $R_{x}^{*} \omega=\omega$, onde $R_{x}^{*}$ é a aplicação dual de $R_{x}$.

Seja $A$ uma álgebra train que satisfaz a identidade (3.1.1). Podemos agora definir o ideal $J$ de $K[\lambda]$ de todos os polinômios $q(\lambda)$ tais que $q\left(R_{x}\right)=0$ para cada $x$ em $A$ de peso 1 . Notemos que $J$ é não vazio já que pelo teorema de Cayley-Hamilton temos que o polinômio de (3.1.1) está em J. Portanto, existe um único polinômio mônico

$$
m(\lambda)=\lambda^{r}+\alpha_{1} \lambda^{r-1}+\cdots+\alpha_{r-1} \lambda+\alpha_{r},
$$

que gera $J$ e é chamado polinômio minimal da álgebra train $A$. Assim, temos que

$$
m\left(R_{x}\right):=R_{x}^{r} y-\alpha_{1} R_{x}^{r-1} y+\cdots+(-1)^{n} \alpha_{r} y=0
$$


para cada $x, y \in A \operatorname{com} \omega(x)=1$. Fazendo $y=x$ em (3.1.2), obtemos a identidade

$$
m\left(R_{x}\right)(x)=x^{r+1}-\alpha_{1} x^{r}+\cdots+\alpha_{r} x=0,
$$

para todo $x \in A$, com $\omega(x)=1$. Dizemos que uma álgebra bárica $A$ é train principal se existe um polinômio não nulo $q(\lambda)$, com $q(0)=0$, tal que $q(x)=0$ para cada elemento $x$ em $A$ de peso 1 . Seja então $A$ uma álgebra train principal. Analogamente à discussão acima, podemos definir o ideal $I$ de $K[\lambda]$ de todos os polinômios $q(\lambda)$ tais que $q(0)=0$ e $q(x)=0$ para cada $x$ em $A$ de peso 1 . Já que $A$ é train principal, o ideal $I$ é não vazio. Portanto, existe um único polinômio mônico

$$
m_{p}(\lambda)=\lambda^{s+1}-\alpha_{1} \lambda^{s}+\cdots+\alpha_{s} \lambda
$$

que gera $I$ e é denominado polinômio minimal principal da álgebra $A$.

O seguinte lema é obvio a partir da discussão acima.

Lema 3.3 Toda álgebra train é álgebra train principal.

A definição acima motiva o seguinte resultado, que é devido a Etherington [7].

Lema 3.4 Se A é uma álgebra train, então cada elemento em $N$ é nilpotente de índice menor ou igual ao grau do polinômio minimal principal de A.

Do lema acima vem que a subálgebra $N=\operatorname{ker} \omega$ de uma álgebra train é sempre nil principal, isto é, todos os seus elementos são nilpotentes principais.

Em geral, algumas das álgebras que surgem a partir de problemas genéticos têm mais propriedades que as álgebras train definidas acima. Isto 
levou Etherington (ver [8]) a definir as álgebras train especiais e a Schafer as álgebras chamadas genéticas.

Uma álgebra bárica $A$ é Gonshor genética se existe uma base, chamada canônica, $\left\{v_{0}, v_{1}, \cdots, v_{n-1}\right\}$ com $\omega\left(v_{0}\right)=1, \omega\left(v_{1}\right)=\cdots=\omega\left(v_{n-1}\right)=0$, satisfazendo

$$
\begin{gathered}
v_{0}^{2}=v_{0}+\sum_{k=1}^{n-1} \lambda_{00 k} v_{k}, \\
v_{0} v_{i}=\sum_{k=i}^{n-1} \lambda_{0 i k} v_{k} \quad(1 \leq i \leq n-1) \\
v_{i} v_{j}=\sum_{k>i, j}^{n-1} \lambda_{i j k} v_{k} \quad(1 \leq i, j \leq n-1) .
\end{gathered}
$$

Note que da primeira equação segue que $\omega\left(v_{0}^{2}\right)=\omega\left(v_{0}\right)=1$ e $\omega\left(v_{k}\right)=0$, para $1 \leq k \leq n-1$. No caso de $A$ ser Gonshor genética temos que $A$ também é Schafer genética no sentido que os coeficientes do polinômio característico de qualquer transformação $T=f\left(R_{x_{1}}, \cdots, R_{x_{t}}\right)$, onde $f\left(\mu_{1}, \cdots, \mu_{t}\right)$ é um polinômio em $t$ associativas não comutativas variáveis sobre $K$, dependem somente das funções $\omega\left(x_{1}\right), \cdots, \omega\left(x_{t}\right)$. De fato, se $A$ é Gonshor genética, para cada $x \in A$ a primeira coordenada de $x$ com respeito à base canônica $\left\{v_{0}, v_{1}, \cdots, v_{n-1}\right\}$ é $\omega(x)$, pois $\omega\left(v_{0}\right)=1$ e $\omega\left(v_{i}\right)=0$ para $i=1, \cdots, n-1$. Então, novamente com respeito à base canônica, a matriz de todo operador linear $R_{x}$ é triangular inferior. Assim, a matriz de $T=f\left(R_{x_{1}}, \cdots, R_{x_{t}}\right)$ é triangular inferior e a entrada $i \times i$ da matriz de $T$ é $f\left(\omega\left(x_{1}\right) \delta_{i i}, \cdots, \omega\left(x_{k}\right) \delta_{i i}\right)$, onde $\delta_{i i}$ representa o elemento na posição $i \times i$ da matriz de $R_{v_{0}}$ com respeito à base canônica. Assim, $A$ é Schafer genética. 
Observamos que nem toda álgebra Schafer genética é Gonshor. O próximo teorema estabelece um critério para a equivalência entre essas estruturas e pode ser encontrado em [26] e [35].

Teorema 3.5 Seja A uma álgebra Schafer genética sobre um corpo $K$ algebricamente fechado. Então A é Gonshor genética.

Observamos da definição que em uma álgebra genética $A$ o barideal $N$ é nilpotente. Uma álgebra bárica $A$ é chamada álgebra train especial se $N$ é nilpotente e todas as potências principais $N^{i}$ são ideais de $A$.

Historicamente, para provar que uma álgebra train especial é uma álgebra train, Etherington [8] mostrou primeiro que toda álgebra train especial possui uma base canônica e, em seguida, que os coeficientes do polinômio característico de toda transformação multiplicação à direita $R_{x}$ são funções apenas de $\omega(x)$. Em [9], além da existência da base canônica, Etherington observa que é requerido também que as potências principais do homomorfismo de peso sejam ideais da álgebra.

Seja $A$ uma $K$-álgebra comutativa de dimensão $n$. Se $F$ é um corpo com $K \subset F$ então podemos definir através do produto tensorial a álgebra $A_{F}=F \otimes_{K} A$, que será uma álgebra comutativa de dimensão $n$ sobre $F$ com produto

$$
(\alpha \otimes x)(\beta \otimes y)=\alpha \beta \otimes x y, \quad \alpha, \beta \in F \quad \text { e } \quad x, y \in A .
$$

Notemos que se $\mathbb{B}=\left\{a_{1}, \cdots, a_{n}\right\}$ é uma base de $A$, então $\mathbb{B}_{F}=\left\{\bar{a}_{1}=\right.$ $\left.1 \otimes a_{1}, \cdots, \bar{a}_{n}=1 \otimes a_{n}\right\}$ será uma base para $A_{F}$. Se $\left\{\gamma_{i j k}\right\}_{i j k=1}^{n}$ são as constantes da estrutura de $A$ com respeito à base $\mathbb{B}$, isto é,

$$
a_{i} a_{j}=\sum_{k} \gamma_{i j k} a_{k} \quad(i, j=1, \cdots, n)
$$


então $\left\{\gamma_{i j k}\right\}_{i j k=1}^{n}$ também são as constantes de estrutura de $A_{F}$ com respeito à base $\mathbb{B}_{F}$.

Seja $f: A \rightarrow A$ uma aplicação linear sobre $K$. Então podemos estender $f$ a uma aplicação $F$-linear $\bar{f}$ de $A_{F}$ em $A_{F}$ definida por

$$
\alpha \otimes x \mapsto \alpha \otimes f(x)
$$

Temos que se $[M]$ é a matriz de $f$ com respeito à base $\mathbb{B}$, então $[M]$ também será a matriz de $\bar{f}$ com respeito à base $\mathbb{B}_{F}$.

Teorema 3.6 Toda álgebra train especial é genética.

Demonstração. Seja $A$ uma álgebra train especial sobre um corpo $K$. Pelas considerações anteriores podemos admitir, sem perda de generalidade, que o corpo $K$ é algebricamente fechado. Sejam $e \in A$ um elemento de peso 1 e $r$ o menor inteiro positivo tal que $0=N^{r}$. Além disso, como $N^{i}$ é um ideal de $A$ temos que $0=N^{r} \subsetneq N^{r-1} \subsetneq N^{r-2} \subsetneq \cdots \subsetneq N^{3} \subsetneq N^{2} \subsetneq N$.

Vamos construir uma base canônica para $A$. Para $i=1, \cdots, r-1$ temos que $e N^{i} \subset N^{i}$, logo podemos definir $\bar{R}_{i}:=N^{i} / N^{i+1} \rightarrow N^{i} / N^{i+1}$ por $\bar{R}_{i}(x+$ $\left.N^{i+1}\right)=x e+N^{i+1}$. Para cada $i=1, \cdots, r-1$, temos que existe uma base $\mathbb{B}_{i}=\left\{\alpha_{i, 1}+N^{i+1}, \cdots, \alpha_{i, s(i)}+N^{i+1}\right\}$ de $N^{i} / N^{i+1}$ tal que $M\left(\bar{R}_{i}, \mathbb{B}_{i}\right)$, a matriz de $\bar{R}_{i}$ com relação à base $\mathbb{B}_{i}$, é triangular inferior. Então a seqüência $\mathbb{B}=\left\{e, \alpha_{1,1}, \cdots, \alpha_{r-1, s(r-1)}\right\}$ é uma base de $A$. Além disso, $M\left(R_{e}, \mathbb{B}\right)$ é triangular inferior e $M\left(R_{y}, \mathbb{B}\right)$ é estritamente triangular, para todo $y \in N$, ou seja, 


$$
\left[R_{e}\right]=\left[\begin{array}{ccccc}
1 & 0 & \cdots & \ldots & 0 \\
* & \alpha_{2} & 0 & \ldots & 0 \\
\vdots & * & \ddots & \ddots & \vdots \\
\vdots & \vdots & \vdots & \alpha_{n-1} & 0 \\
* & * & \ldots & * & \alpha_{n}
\end{array}\right] \text { e }\left[R_{y}\right]=\left[\begin{array}{ccccc}
0 & 0 & \cdots & \cdots & 0 \\
* & 0 & 0 & \cdots & 0 \\
\vdots & * & \ddots & \ddots & \vdots \\
\vdots & \vdots & \vdots & 0 & 0 \\
* & * & \cdots & * & 0
\end{array}\right]
$$

Assim, para todo $x=\omega(x) e+y \in A$, com $y \in N$, temos

$$
\omega(x)\left[R_{e}\right]+\left[R_{y}\right]=\left[R_{x}\right]=\left[\begin{array}{ccccc}
\omega(x) & 0 & \cdots & \cdots & 0 \\
* & \omega(x) \alpha_{2} & 0 & \cdots & 0 \\
\vdots & * & \ddots & \ddots & \vdots \\
\vdots & \vdots & \vdots & \ddots & 0 \\
* & * & \cdots & * & \omega(x) \alpha_{n}
\end{array}\right] .
$$

Agora seja $f\left(\mu_{1}, \cdots, \mu_{t}\right) \in K\left[\mu_{1}, \cdots, \mu_{t}\right]$ uma forma polinomial nas indeterminadas $\lambda_{1}, \cdots, \lambda_{t}$ tal que $f(0, \cdots, 0)=0$. Então, para cada $x_{1}, x_{2}, \cdots, x_{t} \in A$ fazendo $\Delta_{f}:=f\left(\omega\left(x_{1}\right), \cdots, \omega\left(x_{t}\right)\right)$, temos que a matriz $[M]$ de $f\left(R_{x_{1}}, \cdots, R_{x_{t}}\right)$ na base $\mathbb{B}$ fica

$$
[M]=\left[\begin{array}{ccccc}
\Delta_{f} & 0 & \cdots & \cdots & 0 \\
* & \Delta_{f} \alpha_{2} & 0 & \cdots & 0 \\
\vdots & * & \ddots & \ddots & \vdots \\
\vdots & \vdots & \vdots & \ddots & 0 \\
* & * & \cdots & * & \Delta_{f} \alpha_{n}
\end{array}\right]
$$

Portanto o polinômio característico de $f\left(R_{x_{1}}, \cdots, R_{x_{t}}\right)$ é dado por

$$
\operatorname{det}\left(\lambda I-f\left(R_{x_{1}}, \cdots, R_{x_{t}}\right)\right)=\prod_{i=1}^{n}\left(\lambda-\Delta_{f} \alpha_{i}\right),
$$

onde $\alpha_{1}=1$, e o resultado está provado. 
Corolário 3.7 Toda álgebra train especial é uma álgebra train.

Observamos agora que nem toda álgebra genética é uma álgebra train especial, sendo tal afirmação verdadeira somente para álgebras de dimensão menor que 4. A seguir, damos um contra-exemplo para está situação.

Contra-exemplo Usando uma base padrão para $\mathbb{R}^{4}$, definimos a álgebra comutativa com produtos $e_{0}^{2}=e_{0}, e_{0} e_{1}=e_{1}, e_{0} e_{2}=e_{3}, e_{1}^{2}=e_{2}$ e os demais iguais a zero. Está é uma álgebra genética, conforme [26]. Mas, $N^{2}=\left[e_{2}\right]$ não é um ideal de $\mathbb{R}^{4}$, pois $e_{0} e_{2}=e_{3}$. Assim, esta não é uma álgebra train especial.

O esquema abaixo sintetiza as relações entre as álgebras discutidas até aqui.



Passaremos agora ao estudo das relações entre estes tipos de álgebras e as Álgebras de Bernstein. 


\section{2 Álgebras de Bernstein e outras álgebras genéticas}

No que segue, estudamos algumas relações entre as álgebras de Bernstein e as álgebras discutidas na seção anterior.

Teorema 3.8 Em uma álgebra de Bernstein as potências principais de $N$ são ideais de $A$.

Demonstração. Seja $e \in I d A$. Já que $N=\operatorname{ker} \omega$ é um ideal de $A$, é suficiente provar que $e\left(N^{k}\right) \subset N^{k}$, para $k \geq 1$. Provaremos por indução sobre $k$ que $e\left(N^{k}\right) \subset N^{k}$. Para $k=1, e N \subseteq N$ é trivial, já que $N$ é ideal de $A$. Seja $k>1$ e vamos assumir, por hipótese de indução, que $e\left(N^{k-1}\right) \subset N^{k-1}$. Dado um elemento $y_{1} y_{2} \in N^{k}$, com $y_{1} \in N$ e $y_{2} \in N^{k-1}$, temos que $e y_{1} \in N$, pois $N$ é ideal e, pela hipótese de indução que, $e y_{2} \in N^{k-1}$. Portanto, usando a identidade (1.1.6), temos que $2 e\left(y_{1} y_{2}\right)=y_{1} y_{2}-4\left(e y_{1}\right)\left(e y_{2}\right) \in N^{k}+$ $(e N)\left(e N^{k-1}\right) \subset N^{k}$.

Do teorema acima e dos resultados provados anteriormente temos o seguinte corolário.

Corolário 3.9 Para uma álgebra de Bernstein A, as seguintes afirmações são equivalentes:
(a) A é álgebra train especial;
(b) A é álgebra genética;
(c) $N$ é nilpotente. 
Estudaremos a seguir as potências principais de elementos do barideal $N$ e da álgebra $A$. Seja $y=u+v \in N$. Então, do Lema 1.16, temos que

$$
\begin{aligned}
& y^{2}=u^{2}+2 u v+v^{2} \\
& y^{3}=u^{2} v+2(u v) v+v^{3}=R_{v}\left(y^{2}\right) \in L
\end{aligned}
$$

e, indutivamente, é fácil ver que

$$
y^{k+1}=R_{v}^{k-1}\left(y^{2}\right) \quad \text { para } \quad k \geq 2,
$$

já que, $U_{e} L=0$ e $L$ é um ideal de $A$.

Seja agora $x=e+u+v \in A$. Então, das identidades (1.1.12), obtemos

$$
\begin{aligned}
& x^{2}=e+\left(u+2 u v+v^{2}\right)+u^{2}, \\
& x^{3}=e+\left(u+2 u v+\frac{1}{2} v^{2}+u^{2} v+2(u v) v+v^{3}\right)+u^{2},
\end{aligned}
$$

$\log 0$

$$
x^{3}-x^{2}=-\frac{1}{2} v^{2}+u^{2} v+2(u v) v+v^{3} \in L,
$$

e, portanto,

$$
\begin{aligned}
\left(x^{3}-x^{2}\right) x & =\left(x^{3}-x^{2}\right) e+\left(x^{3}-x^{2}\right) v \\
& =\frac{1}{2}\left(x^{3}-x^{2}\right)+R_{v}\left(x^{3}-x^{2}\right)
\end{aligned}
$$

donde, $\left(x^{3}-x^{2}\right)\left(x-\frac{1}{2}\right)=R_{v}\left(x^{3}-x^{2}\right)$.

Novamente por indução em $k$, é fácil ver que

$$
\left(R_{x}-\frac{1}{2} I d\right)^{k}\left(x^{3}-x^{2}\right)=R_{v}^{k}\left(x^{3}-x^{2}\right),
$$


para $k \geq 1$. Aplicando o Lema 1.16, temos

$$
\begin{aligned}
R_{x}\left(R_{x}-\frac{1}{2} I d\right)^{k-1}\left(x^{3}-x^{2}\right)= & e R_{v}^{k-1}\left(x^{3}-x^{2}\right)+u R_{v}^{k-1}\left(x^{3}-x^{2}\right) \\
& +v R_{v}^{k-1}\left(x^{3}-x^{2}\right) \\
= & \frac{1}{2} R_{v}^{k-1}\left(x^{3}-x^{2}\right)+v R_{v}^{k-1}\left(x^{3}-x^{2}\right) \\
= & \frac{1}{2}\left(R_{x}-\frac{1}{2} I d\right)^{k-1}\left(x^{3}-x^{2}\right)+R_{v}^{k}\left(x^{3}-x^{2}\right)
\end{aligned}
$$

e $\operatorname{assim}\left(R_{x}-\frac{1}{2} I d\right)^{k}\left(x^{3}-x^{2}\right)=R_{v}^{k}\left(x^{3}-x^{2}\right)$.

De interesse especial é a relação entre as álgebras de Bernstein e as álgebras train e train principal. As considerações e resultados que seguem visam este estudo.

Teorema 3.10 Se uma álgebra de Bernstein A de tipo $(r, s)$ é uma álgebra train, então as raízes train são $1, \frac{1}{2}$ e 0 , com multiplicidades $1, r-1$ es, respectivamente.

Demonstração. Seja $e$ um idempotente. Com respeito a decomposição $K e \oplus U_{e} \oplus V_{e}$ temos que, $R_{e}(e)=e, R_{e}(u)=\frac{1}{2} u$ e $R_{e}(v)=0$. Então, o polinômio característico de $R_{e}$ é dado por

$$
\operatorname{det}\left(\lambda I d-R_{e}\right)=(\lambda-1)\left(\lambda-\frac{1}{2}\right)^{r-1} \lambda^{s}
$$

donde segue a demonstração.

Sejam $x \in A$ um elemento de peso 1 e $e=x^{2}$ idempotente de $A$. Existem $\bar{u} \in U_{e}$ e $\bar{v} \in V_{e}$, únicos, tais que $x=e+\bar{u}+\bar{v}$. Já que $x^{2}=e$, temos $\bar{u}^{2}=0$ e $\bar{u}+2 \bar{u} \bar{v}+\bar{v}^{2}=0$.

Vamos agora analisar a matriz do operador $R_{x}: A \rightarrow A$. Seja $\vec{R}_{x}$ a restrição de $R_{x}$ ao barideal $N$. A identidade $2 x^{2}(x y)=x y$, com $y \in N$, 
pode ser escrita na forma $M_{e}\left(\bar{R}_{x}\right)=\bar{R}_{x}$, onde $M_{e}$ é o operador projeção $M_{e}: N \rightarrow N$ definido por $M_{e}(y)=2 e y$.

Da identidade $M_{e}\left(\bar{R}_{x}\right)=\bar{R}_{x}$ vem que

$$
\operatorname{Im} \bar{R}_{x} \subset \operatorname{Im} M_{e}=U_{e}
$$

e daí, as $V_{e}$-componentes de $\bar{R}_{x}(u)$ e $\bar{R}_{x}(v)$ são nulas para todo $u \in U_{e}$ e $v \in V_{e}$. Em particular, $\bar{u} \in L$ e $\bar{R}_{x}(u)=e u+\bar{v} u=\frac{1}{2} u+\bar{R}_{\bar{v}}(u)$, e $\bar{R}_{x}(v)=e v+\bar{u} v+\bar{v} v=(\bar{u}+\bar{v}) v \in U_{e}$.

A representação matricial de $R_{x}$ com respeito a uma decomposição $A=$ $K e \oplus V_{e} \oplus U_{e}$ fica na forma

$$
\left[R_{x}\right]=\left[\begin{array}{ccc}
1 & 0 & 0 \\
\square & 0 & 0 \\
\square & \square & {\left[\frac{1}{2} I d+\bar{R}_{\bar{v}}\right]}
\end{array}\right],
$$

onde $\square$ representam blocos matriciais que são irrelevantes para o cálculo do polinômio característico de $R_{x}$ e $\left[\frac{1}{2} I d+\bar{R}_{\bar{v}}\right]$ a matriz da restrição de $\frac{1}{2} I d_{\mathbf{u}}+\bar{R}_{\bar{v}}$ ao subespaço $U_{e}$.

Da discussão acima temos o seguite resultado.

Lema 3.11 Sejam A uma álgebra de Bernstein de tipo $(r, s)$ e $x \in A$ um elemento de peso 1. Se $e=x^{2}$ e $x=e+\bar{u}+\bar{v}$ com $\bar{u} \in U_{e}$ e $\bar{v} \in V_{e}$, então o polinômio característico de $R_{x}$ é dado por

$$
\operatorname{det}\left(\lambda I d-R_{x}\right)=(\lambda-1) \lambda^{s} Q\left(\lambda-\frac{1}{2}\right),
$$

onde $Q(\lambda)$ é o polinômio característico da restrição de $R_{\bar{v}}$ ao subespaço $U_{e}$.

Observe ainda que $x-x^{2}=\bar{u}+\bar{v}, \bar{u}=M_{e}(\bar{u}+\bar{v})=2 x^{2}\left(x-x^{2}\right)=2 x^{3}-2 x^{2}$, e $\bar{v}=x+x^{2}-2 x^{3}$. O próximo teorema estabelece uma condição para que 
uma álgebra de Bernstein seja uma álgebra train. O resultado é devido a Lyubich [26].

Teorema 3.12 Para que uma álgebra de Bernstein A seja uma álgebra train é necessário e suficiente que o operador $R_{y}$ seja nilpotente para todo $y \in N$.

Demonstração. Em toda álgebra train, $R_{y}$ é nilpotente em $N$, para todo $y \in N$. Por outro lado, vamos assumir que $R_{y}$ seja nilpotente para todo $y \in N$. Se $x$ é um elemento de peso 1 então, pelo lema anterior,

$$
\operatorname{det}\left(\lambda I d-R_{x}\right)=(\lambda-1) \lambda^{s} Q\left(\lambda-\frac{1}{2}\right),
$$

onde $Q(\lambda)$ é o polinômio característico de $R_{\bar{v}}$ em $U_{e}$, sendo $e=x^{2}, x=$ $e+\bar{u}+\bar{v} \operatorname{com} \bar{u} \in U_{e}$ e $\bar{v} \in V_{e}$. Pela hipótese $R_{\bar{v}}$ é nilpotente, logo $Q(\lambda)=\lambda^{r-1}$ e portanto

$$
\operatorname{det}\left(\lambda I d-R_{x}\right)=(\lambda-1) \lambda^{s}\left(\lambda-\frac{1}{2}\right)^{r-1}
$$

Conseqüentemente o polinômio característico de $R_{x}$ independe do elemento $x$ de peso 1 e assim a álgebra é train.

O próximo teorema sintetiza as relações discutidas até aqui.

Teorema 3.13 Para uma álgebra de Bernstein $A=K e \oplus U_{e} \oplus V_{e}$, as seguintes afirmações são equivalentes:

(a) A é álgebra train;

(b) A é álgebra train principal;

(c) $N$ é nil;

(d) $R_{v}: L \rightarrow L$ é nilpotente para todo $v \in V_{e}$;

(e) $R_{w}: U_{f} \rightarrow U_{f}$ é nilpotente para todo $f$ idempotente $e w \in V_{f}$. 
Demonstração. (a) $\Rightarrow$ (b) Segue do Lema 3.3.

(b) $\Rightarrow$ (c) É imediato a partir da definição de álgebra train principal.

(c) $\Rightarrow$ (d) Seja $k$ tal que $y^{k}=0$ para todo $y \in N$. Se $\bar{u} \in L$ e $v \in V_{e}$ então definimos $y=\bar{u}+v$ e, por (3.2.1) temos que

$$
0=y^{k}=R_{v}^{k-2}\left(y^{2}\right)=R_{v}^{k-2}\left(2 \bar{u} v+v^{2}\right)=2 R_{v}^{k-1}(\bar{u})+v^{k}=2 R_{v}^{k-1}(\bar{u}) .
$$

Logo, podemos afirmar que $R_{v}^{k-1}(L)=0$ para cada $v \in V_{e}$.

(d) $\Rightarrow$ (e) Vamos assumir que $R_{v}$ em $L$ é nilpotente de índice menor ou igual a $k-1$, para todo $v \in V_{e}$ e sejam agora $f$ um idempontente de $A$ e $w \in V_{f}$. Provaremos que $R_{w}$ em $U_{f}$ é nilpotente de índice menor ou igual a $k+1$. Se $u \in U_{f}$ então $w(w u) \in L, \operatorname{logo}$

$$
R_{w}^{k+1}(u)=R_{w}^{k-1}(w(w u)) \in R_{w}^{k-1}(L)=0,
$$

e assim $R_{w}$ é nilpotente em $U_{f}$.

(e) $\Rightarrow$ (a) Sejam $x \in A$ de peso $1, e=x^{2}, \bar{u} \in U_{e}$ e $\bar{v} \in V_{e}$ tais que $x=e+\bar{u}+\bar{v}$. Pelo Lema 3.11 temos que

$$
\operatorname{det}\left(\lambda I d-R_{x}\right)=(\lambda-1) \lambda^{s} Q\left(\lambda-\frac{1}{2}\right),
$$

onde $Q(\lambda)$ é o polinômio característico de $R_{\bar{v}}$ sobre $U_{e}$. Agora, por hipótese, $R_{\bar{v}}$ é nilpotente, $\log Q Q(\lambda)=\lambda^{r-1}$ e assim,

$$
\operatorname{det}\left(\lambda I d-R_{x}\right)=(\lambda-1) \lambda^{s}\left(\lambda-\frac{1}{2}\right)^{r-1},
$$

e o resultado está provado.

O próximo resultado estabelece uma relação entre as álgebras de Bernstein e as álgebras train especiais. O resultado é devido à M.Ouattara (ver [31]). 
Teorema 3.14 Toda álgebra de Bernstein train de dimensão menor ou igual a 5 é uma álgebra train especial.

Demonstração. Suponhamos que $A$ é uma álgebra de Bernstein train de dimensão 5. Então a dimensão de $N$ é 4 e $x^{5}=0$, para todo $x \in N$. Se o nil índice de $N$ é 5 , existe $0 \neq x \in N$ tal que $\left\{x, x^{2}, x^{3}, x^{4}\right\}$ seja uma base para $N$. Usando a identidade (1.1.2), a tabela de multiplicação de $N$ cumpre $x^{i} x^{j}=0$ para $i, j \geq 2$. Assim, $N^{2}=\left\langle x^{2}, x^{3}, x^{4}\right\rangle, N^{3}=\left\langle x^{3}, x^{4}\right\rangle, N^{4}=\left\langle x^{4}\right\rangle$ e $N^{5}=0$. Portanto, $N$ é nilpotente principal de índice 5 e assim $A$ é uma álgebra train especial. Agora, se o nil índice de $N$ é inferior a 5 , da identidade (1.1.2), para $i, j \geq 2$, segue que a subálgebra $N$ é de potências associativas e, conforme [11], toda nil álgebra comutativa, de potências associativas e dimensão 4 é nilpotente principal. Logo $A$ é uma álgebra train especial. 


\section{Capítulo 4}

\section{Solubilidade do barideal}

O objetivo deste capítulo é estudar a nilpotência e a solubilidade do barideal de uma álgebra de Bernstein. O estudo dessa estrutura tem início a partir dos resultados obtidos por Lyubich [26]. Em especial, Lyubich demonstrou que uma álgebra de Bernstein de dimensão finita é genética se, e somente se seu núcleo é nilpotente principal e A. Grishkov demonstrou em [15] que toda álgebra de Bernstein nuclear de dimensão finita é genética. Mais tarde, C. Martínez, em [27], e J.C. Gutierréz, em [10], provaram que se uma álgebra de Bernstein nuclear é gerada por $r$ elementos, então $\operatorname{ker} \omega^{2 r+2}=0$ e finalmente J.Bernad em [2] que $\operatorname{ker} \omega^{r+4}=0$. Por outro lado, I.R.Hentzel, D.P.Jacobs, P.Sverchkov e L.A.Peresi, em [19], demonstraram que o núcleo de uma álgebra de Bernstein é solúvel de grau 4. Neste trabalho demonstraremos que em uma álgebra de Bernstein o barideal $N$ cumpre $\left(N^{2}\right)^{5}=0$ e $N^{[3]}=0$. Estes resultados são apresentados em [2].

No que segue, para $b, a_{1}, \ldots, a_{n} \in N$, usaremos as notações:

I. $\quad \mathrm{a}_{n}:=a_{1} a_{2} \ldots a_{n}$, onde $a_{1} a_{2} \ldots a_{n}:=a_{1} R_{a_{2}} \ldots R_{a_{n}} ;$

II. $b \mathbf{a}_{n}:=b a_{1} a_{2} \ldots a_{n}=b R_{a_{1}} \ldots R_{a_{n}} \mathrm{e}$, 
III. Se $\sigma \in S_{n}$, então $\mathbf{a}_{\sigma(n)}:=a_{\sigma(1)} a_{\sigma(2)} \ldots a_{\sigma(n)}$, onde $S_{n}$ representa o grupo simétrico de $n$ elementos.

Ao longo deste capítulo $A=K e \oplus U \oplus V$ será uma álgebra de Bernstein sobre o corpo $K$, e um idempotente fixo, e $N$ representará o barideal da álgebra. Assim $N=U \oplus V$, onde $U$ e $V$ são as componentes da decomposição de Peirce de $A$ com respeito ao idempotente $e$. Como usualmente $u$ e $u_{1}, \ldots, u_{n}$ são elementos em $U$, e $v$ e $v_{1}, \ldots, v_{n}$ elementos em $V$.

\subsection{Nilpotência e Solubilidade do barideal}

Analisaremos a nilpotência e a solubilidade do barideal $N$. No que segue, estabeleceremos algumas propriedades do produto nas álgebras de Bernstein que serão úteis na demonstração dos resultados descritos acima.

Lema 4.1 Se A é uma álgebra de Bernstein-Jordan, então temos que

$$
N^{k}=N^{(k)}
$$

Demonstração. Primeiro provaremos por indução sobre o mínimo de $i, j$ que $N^{i} N^{j} \subset N^{i+j}$. Para $i=1$ o resultado é trivial. Seja então $1<i \leq j$ e suponhamos que $N^{s} N^{t}=N^{s+t}$, para todo $s, t$ com $1 \leq s<i$ e $s<t$. Por (1.2.6) vem que

$$
\begin{aligned}
\left(\mathbf{a}_{i-1} a_{i}\right)\left(\mathbf{b}_{j}\right) & =-\left(\mathbf{a}_{i-1} \mathbf{b}_{j}\right) a_{i}-\mathbf{a}_{i-1}\left(\mathbf{b}_{j} a_{i}\right) \\
& \in\left(N^{i-1} N^{j}\right) N+N^{i-1} N^{j+1} \subset N^{i+j},
\end{aligned}
$$

de onde segue a igualdade desejada. 
Agora mostraremos que $N^{k}=N^{(k)}$. Para $k=1$, o lema é trivial. Seja $k>1$ e suponhamos que $N^{i}=N^{(i)}$, para $1 \leq i<k$. Então segue que

$$
N^{(k)}=\sum_{i+j=k} N^{(i)} N^{(j)}=\sum_{i+j=k} N^{i} N^{j} \subset N^{k} .
$$

A outra inclusão é trivial.

Lema 4.2 Em uma álgebra de Bernstein-Jordan temos que

$$
u \mathbf{v}_{k}=(-1)^{\sigma} u \mathbf{v}_{\sigma(k)}
$$

para cada $\sigma \in S_{k}$, onde $(-1)^{\sigma}$ representa o sinal de $\sigma$. Em particular, temos que $\left(u \mathbf{v}_{k}\right) v_{i}=0$ para todo $i$ com $1 \leq i \leq k$.

Demonstração. É suficiente provar que

$$
\left(\ldots\left(\left(\left(u \mathbf{v}_{i-1}\right) v_{i}\right) v_{i+1}\right) \ldots\right) v_{k}=-\left(\ldots\left(\left(\left(u \mathbf{v}_{i-1}\right) v_{i+1}\right) v_{i}\right) \ldots\right) v_{k}
$$

para todo $i$ com $1 \leq i<k$. E esta igualdade segue da identidade (1.2.7), já que $u \mathbf{v}_{i-1} \in U$.

O próximo lema mostra que o produto $u_{1} v_{1} v_{2} \cdots v_{k} u_{2}$ é simétrico ou antisimétrico em $u_{1}$ e $u_{2}$, dependendo do número de elementos de $V$ entre eles. Está idéia será fundamental para a demonstração dos resultados desse capítulo.

Lema 4.3 Sejam A uma álgebra de Bernstein-Jordan, $u_{1}, u_{2} \in U \quad e$ $v_{1}, \ldots, v_{k} \in V$. Então:

(a) se $k \equiv 1,2(\bmod 4), u_{1} \mathbf{v}_{k} u_{2}+u_{2} \mathbf{v}_{k} u_{1}=0$;

(b) se $k \equiv 0,3(\bmod 4), u_{1} \mathbf{v}_{k} u_{2}-u_{2} \mathbf{v}_{k} u_{1}=0$. 
Demonstração. Provaremos por indução em $k$ que

$$
u_{1} \mathbf{v}_{k} u_{2}=(-1)^{\frac{k(k+1)}{2}} u_{2} \mathbf{v}_{k} u_{1}
$$

Para $k=1$ a igualdade é trivial. Seja $k>1$ e assumamos a igualdade para todo $i$ com $1 \leq i<k$. Então temos que

$$
\begin{aligned}
u_{1} \mathbf{v}_{k} u_{2} & =u_{1} \mathbf{v}_{k-1} v_{k} u_{2} \\
& =-\left(u_{1} \mathbf{v}_{k-1}\right)\left(u_{2} v_{k}\right) \\
& =(-1)(-1)^{\frac{(k-1) k}{2}} u_{2} v_{k} \mathbf{v}_{k-1} u_{1} \\
& =(-1)^{\frac{(k-1) k}{2}+1+k-1} u_{2} \mathbf{v}_{k} u_{1} \\
& =(-1)^{\frac{(k-1) k+2 k}{2}} u_{2} \mathbf{v}_{k} u_{1} \\
& =(-1)^{\frac{k(k+1)}{2}} u_{2} \mathbf{v}_{k} u_{1} .
\end{aligned}
$$

Corolário 4.4 Sejam $A=K e \oplus U_{e} \oplus V_{e}$ uma álgebra de BernsteinJordan, $k \equiv 1,2(\bmod 4), \quad t \equiv 0,3(\bmod 4), a_{1}, \cdots, a_{r}, b_{1}, \cdots, b_{s} \in N$, $v_{1}, \cdots, v_{k}, w_{1}, \cdots, w_{t} \in V_{e}, \quad e \alpha \in K-\{0\} . \quad$ Se $x \mathbf{v}_{k} y \mathbf{a}_{r}=\alpha x \mathbf{w}_{t} y \mathbf{b}_{s}$ para todo $x, y \in U_{e}$, então

$$
x \mathbf{v}_{k} y \mathbf{a}_{r}=0 \quad e \quad x \mathbf{w}_{t} y \mathbf{b}_{s}=0,
$$

para todo $x, y \in U_{e}$.

Demonstração. Trocando $x$ e $y$ na identidade que estamos assumindo e usando o Lema 4.3 obtemos o resultado desejado.

O próximo lema apresenta o menor valor que a dimensão do subespaço $U$ pode assumir. O resultado é devido a Cortéz e Montaner (ver [5]). 
Lema 4.5 Se A é uma álgebra de Bernstein-Jordan e existem $u_{1}, u_{2} \in U$ e $v_{1}, \ldots, v_{k} \in V$ tais que $u_{1} \mathbf{v}_{k} u_{2} \neq 0, \operatorname{com} k \equiv 1,2(\bmod 4)$, então $\operatorname{dim} U \geq 2^{k+1}$.

Demonstração. Vejamos que a seqüência formada pelos elementos definidos por $u_{j} v_{i_{1}} \ldots v_{i_{t}}$, onde $j \in\{1,2\}, 0 \leq t \leq k$ e $1 \leq i_{1}<\ldots<i_{t} \leq k$, é linearmente independente. Seja

$$
\sum \lambda_{i_{1} \ldots i_{t}} u_{1} v_{i_{1}} \cdots v_{i_{t}}+\sum \mu_{i_{1} \ldots i_{t}} u_{2} v_{i_{1}} \cdots v_{i_{t}}=0
$$

uma combinação linear dos elementos igual a zero. Multiplicando a igualdade sucessivamente por $v_{1}, \ldots, v_{k}$ e usando o Lema 4.2 obtemos

$$
\lambda_{0} u_{1} v_{1} v_{2} \cdots v_{k}+\mu_{0} u_{2} v_{1} v_{2} \cdots v_{k}=0
$$

Se multiplicarmos agora por $u_{1}$ obtemos $\mu_{0} u_{2} \mathbf{v}_{k} u_{1}=0$, logo $\mu_{0}=0$ e, se multiplicarmos por $u_{2}$ obtemos analogamente que $\lambda_{0}=0$. Para cada $r$ com $1 \leq r \leq k$ obtemos, multiplicando (4.1.1) sucessivamente por $v_{1}, \ldots, v_{r-1}, v_{r+1}, \ldots, v_{k}$ e aplicando o Lema 4.2 , que

$$
\lambda_{r} u_{1} v_{r} v_{1} \cdots v_{r-1} v_{r+1} \cdots v_{k}+\mu_{r} u_{2} v_{r} v_{1} \cdots v_{r-1} v_{r+1} \cdots v_{k}=0
$$

Se multiplicarmos a identidade acima por $u_{1}$ obtemos $\mu_{r} u_{2} \mathbf{v}_{k} u_{1}=0$, logo $\mu_{r}=0$ e, se multiplicarmos por $u_{2}$ obtemos, analogamente, que $\lambda_{r}=0$.

Agora, para cada par $(r, s)$ com $1 \leq r<s \leq k$, obtemos multiplicando (4.1.1) sucessivamente por $v_{1}, \ldots, v_{r-1}, v_{r+1}, \ldots, v_{s-1}, v_{s+1}, \ldots, v_{k}$, e aplicando o Lema 4.3, temos que

$$
\begin{aligned}
& \lambda_{r s} u_{1} v_{r} v_{s} v_{1} \cdots v_{r-1} v_{r+1} \cdots v_{s-1} v_{s+1} \cdots v_{k}+ \\
& \mu_{r s} u_{2} v_{r} v_{r} v_{1} \cdots v_{r-1} v_{r+1} \cdots v_{s-1} v_{s+1} \cdots v_{k}=0 .
\end{aligned}
$$


Novamente, se multiplicarmos a última identidade por $u_{1}$ obtemos $\mu_{r s} u_{2} \mathbf{v}_{k} u_{1}=0$, logo $\mu_{r s}=0$ e, se multiplicarmos por $u_{2}$ obtemos, analogamente, que $\lambda_{r s}=0$.

Continuando com o processo acima, segue que cada coeficiente $\lambda_{i_{1} \cdots i_{t}}$ e $\mu_{i_{1} \cdots i_{t}}$ é zero e portanto, a seqüência definida acima é linearmente independente e, uma vez que há $2^{k+1}$ elementos, o resultado está provado.

Os resultados que seguem destacam o produto de elementos de $U$ e $V$ em uma álgebra de Bernstein-Jordan, e serão fundamentais na demonstração de $\left(N^{2}\right)^{5}=0$.

Primeiro observe que, se $A$ é uma álgebra de Bernstein-Jordan, temos

$$
x \mathbf{v}_{t} x^{2}=(-1)^{t} x x^{2} \mathbf{v}_{t}=(-1)^{t} x^{3} \mathbf{v}_{t}=0
$$

para todo $x \in U$. Linearizando a identidade anterior segue imediatamente que

$$
u_{1} \mathbf{v}_{t}\left(u_{2} u_{3}\right)+u_{2} \mathbf{v}_{t}\left(u_{1} u_{3}\right)+u_{3} \mathbf{v}_{t}\left(u_{1} u_{2}\right)=0
$$

para $u_{1}, u_{2}, u_{3} \in U$

Lema 4.6 Sejam A uma álgebra de Bernstein-Jordan, $x, y, u \in U e$ $v_{1}, \cdots, v_{t} \in V$. Então:

(a) $x \mathbf{v}_{t} x u=u \mathbf{v}_{t} x^{2}$, se $t \equiv 0,3(\bmod 4)$;

(b) $x y^{2} \mathbf{v}_{t} x=y x^{2} \mathbf{v}_{t} y$. 
Demonstração. No item (a) temos que

$$
\begin{aligned}
x \mathbf{v}_{t} x u & =-x \mathbf{v}_{t} u x-x \mathbf{v}_{t}(x u) \\
& =-u \mathbf{v}_{t} x x+\frac{1}{2} u \mathbf{v}_{t} x^{2} \\
& =\frac{1}{2} u \mathbf{v}_{t} x^{2}+\frac{1}{2} u \mathbf{v}_{t} x^{2} \\
& =u \mathbf{v}_{t} x^{2} .
\end{aligned}
$$

Para o item (b) observe que

$$
\begin{aligned}
x y^{2} \mathbf{v}_{t} x & =-2 y(x y) \mathbf{v}_{t} x \\
& =2(-1)^{t+1} y \mathbf{v}_{t}(x y) x \\
& =2(-1)^{t} y \mathbf{v}_{t}(x(x y)) \\
& =(-1)^{t+1} y \mathbf{v}_{t}\left(y x^{2}\right) \\
& =(-1)^{t} y \mathbf{v}_{t} x^{2} y \\
& =y x^{2} \mathbf{v}_{t} y
\end{aligned}
$$

Portanto, o lema está provado.

Linearizando as igualdades dos itens (a) e (b) do lema acima em $x$ e $y$, obtemos

$$
\begin{aligned}
& u_{1} \mathbf{v}_{t} u_{2} u_{3}=u_{3} \mathbf{v}_{t}\left(u_{1} u_{2}\right), \text { para } t \equiv 0,3(\bmod 4) \\
& y_{1} y_{2} x_{1} \mathbf{v}_{2} x_{2}=x_{1} x_{2} y_{1} \mathbf{v}_{2} y_{2} .
\end{aligned}
$$

Corolário 4.7 Sejam A uma álgebra de Bernstein-Jordan, $x, y, u \in U e$ $v_{1}, v_{2} \in V$. Então

$$
x y^{2} \mathbf{v}_{2} x u=0 .
$$


Demonstração. Observe primeiro que

$$
\begin{aligned}
& x y^{2} \mathbf{v}_{2} x u=-x y^{2} \mathbf{v}_{2} u x-x y^{2} \mathbf{v}_{2}(x u) \\
& \stackrel{4.1 .4}{=}-2 x y^{2} \mathbf{v}_{2}(x u) \\
&=2 x(x u) y^{2} \mathbf{v}_{2} \\
&=-u x^{2} y^{2} \mathbf{v}_{2}
\end{aligned}
$$

Por outro lado,

$$
\begin{aligned}
x y^{2} \mathbf{v}_{2} x u \stackrel{4.1 .5}{=} & y x^{2} \mathbf{v}_{2} y u \\
& =-y x^{2} \mathbf{v}_{2} u y-y x^{2} \mathbf{v}_{2}(y u) \\
& \stackrel{4.1 .4}{=}-2 y x^{2} \mathbf{v}_{2}(y u) \\
& =2 y(y u) x^{2} \mathbf{v}_{2} \\
& =-u y^{2} x^{2} \mathbf{v}_{2} \\
& =u x^{2} y^{2} \mathbf{v}_{2} .
\end{aligned}
$$

Donde segue a identidade.

Linearizando a igualdade do corolário anterior em $x$ e $y$ temos que

$$
u_{1} u_{2} u_{3} \mathbf{v}_{2} u_{4} u_{5}=0
$$

Lema 4.8 Para todo $u_{1}, u_{2}, u_{3} \in U$ e $v_{1}, v_{2}, v_{3} \in V$ em uma álgebra de Bernstein-Jordan, temos

$$
u_{1} \mathbf{v}_{2} u_{2} u_{3} v_{3}=-u_{1} v_{3} u_{2} u_{3} \mathbf{v}_{2}
$$

Demonstração. Da identidade (1.2.6) temos que

$$
(u v)\left(u_{3} v_{3}\right) u_{2}+(u v) u_{2}\left(u_{3} v_{3}\right)+\left(u_{3} v_{3}\right) u_{2}(u v)=0
$$


Daí segue que

$$
u v v_{3} u_{3} u_{2}+u v u_{2} u_{3} v_{3}+u_{3} v_{3} u_{2} u v=0
$$

Trocando $u$ por $u_{1} v_{1}$ e $v$ por $v_{2}$ temos

$$
u_{1} \mathbf{v}_{3} u_{3} u_{2}+u_{1} \mathbf{v}_{2} u_{2} u_{3} v_{3}-u_{3} v_{3} u_{2} u_{1} \mathbf{v}_{2}=0
$$

Permutando $u_{1}, u_{2}, u_{3}$ em (4.1.8) e somando as identidades vem que

$$
\begin{aligned}
& u_{1} \mathbf{v}_{\mathbf{3}} u_{3} u_{2}+u_{1} \mathbf{v}_{\mathbf{2}} u_{2} u_{3} v_{3}-u_{3} v_{3} u_{2} u_{1} \mathbf{v}_{\mathbf{2}}+ \\
& u_{1} \mathbf{v}_{\mathbf{3}} u_{2} u_{3}+u_{1} \mathbf{v}_{\mathbf{2}} u_{3} u_{2} v_{3}-u_{2} v_{3} u_{3} u_{1} \mathbf{v}_{\mathbf{2}}+ \\
& u_{3} \mathbf{v}_{\mathbf{3}} u_{2} u_{1}+u_{3} \mathbf{v}_{\mathbf{2}} u_{1} u_{2} v_{3}-u_{2} v_{3} u_{1} u_{3} \mathbf{v}_{\mathbf{2}}=0 .
\end{aligned}
$$

Por (4.1.4) a soma dos termos da primeira coluna da identidade acima é zero, isto é,

$$
u_{1} \mathbf{v}_{\mathbf{3}} u_{3} u_{2}+u_{1} \mathbf{v}_{\mathbf{3}} u_{2} u_{3}+u_{3} \mathbf{v}_{\mathbf{3}} u_{2} u_{1}=0
$$

já que $u_{i} \mathbf{v}_{\mathbf{3}} u_{j} u_{k}=u_{k} \mathbf{v}_{\mathbf{3}}\left(u_{i} u_{j}\right)=-u_{k}\left(u_{i} u_{j}\right) \mathbf{v}_{\mathbf{3}}$ para todo $i, j, k \in\{1,2,3\}$.

Pelo Lema 4.3 segue que a soma dos dois últimos termos da segunda coluna também é zero,

$$
u_{1} \mathbf{v}_{2} u_{3} u_{2} v_{3}+u_{3} \mathbf{v}_{2} u_{1} u_{2} v_{3}=0
$$

Analogamente, a soma dos dois primeiros termos da terceira coluna é zero, ou seja, $u_{3} v_{3} u_{2} u_{1} \mathbf{v}_{2}+u_{2} v_{3} u_{3} u_{1} \mathbf{v}_{2}=0$, de maneira que

$$
u_{1} \mathbf{v}_{2} u_{2} u_{3} v_{3}+u_{1} v_{3} u_{2} u_{3} \mathbf{v}_{2}=0
$$


Corolário 4.9 Sejam $A$ uma álgebra de Bernstein-Jordan, $u_{1}, u_{2}, u_{3} \in U$ e $v_{1}, v_{2}, v_{3}, v_{4} \in V$. Então

$$
u_{1} \mathbf{v}_{3} u_{2} u_{3} v_{4}=0, \quad u_{1} \mathbf{v}_{2} u_{2} u_{3} v_{3} v_{4}=0
$$

Demonstração. Trocando $u_{1}$ por $u_{1} v$ no lema anterior e combinando com o Corolário 4.4 obtemos o resultado.

Proposição 4.10 Qualquer produto de três elementos de U e quatro elementos de $V$ em uma álgebra de Bernstein-Jordan é nulo.

Demonstração. Aplicando o corolário anterior e o fato do produto de dois elementos de $V$ ser nulo, para provar a proposição é suficiente mostrar que

$$
u_{1} \mathbf{v}_{4} u_{2} u_{3}=0 \quad u_{1} v_{1} u_{2} u_{3} v_{2} v_{3} v_{4}=0, \quad u_{1} u_{2} u_{3} \mathbf{v}_{4}=0
$$

para todo $u_{1}, u_{2}, u_{3} \in U$ e $v_{1}, v_{2}, v_{3}, v_{4} \in V$. Observe que

$$
\begin{array}{rll}
u_{1} \mathbf{v}_{4} u_{2} u_{3} & \stackrel{4.6}{=} & u_{3} \mathbf{v}_{4}\left(u_{1} u_{2}\right) \\
& \stackrel{1.2 .7}{=}-u_{3} \mathbf{v}_{3}\left(u_{1} u_{2}\right) v_{4} \\
& \stackrel{1.2 .6}{=} u_{3} \mathbf{v}_{3} u_{1} u_{2} v_{4}+u_{3} \mathbf{v}_{3} u_{2} u_{1} v_{4}=0,
\end{array}
$$

pelo corolário anterior. Para a segunda identidade temos que

$$
u_{1} v_{1} u_{2} u_{3} v_{2} v_{3} v_{4} \stackrel{4.8}{=}-u_{1} v_{2} v_{3} u_{2} u_{3} v_{1} v_{4}=0
$$

Finalmente,

$$
\begin{aligned}
& u_{1} u_{2} u_{3} \mathbf{v}_{4} \stackrel{1.2 .7}{=} u_{3} \mathbf{v}_{4}\left(u_{1} u_{2}\right) \\
& \stackrel{1.2 .6}{=}-u_{3} \mathbf{v}_{4} u_{1} u_{2}-u_{3} \mathbf{v}_{4} u_{2} u_{1}=0 .
\end{aligned}
$$


Proposição 4.11 Qualquer produto de quatro elementos de U e três elementos de $V$ em uma álgebra de Bernstein-Jordan é nulo.

Demonstração. Do Lema 4.8 temos que

$$
\begin{aligned}
0 & =u_{1} v_{1} u_{2} u_{3} v_{2} v_{3} u_{4}+u_{1} v_{2} v_{3} u_{2} u_{3} v_{1} u_{4} \\
& =u_{3}\left(u_{1} v_{1} u_{2}\right) v_{3} u_{4}+u_{3}\left(u_{1} v_{2} v_{3} u_{2}\right) u_{4}
\end{aligned}
$$

e agora pelo Corolário 4.4 segue que cada termo é zero. Assim

$$
u_{1} v_{1} u_{2} u_{3} v_{2} v_{3} u_{4}=0 \quad \text { e } \quad u_{1} v_{2} v_{3} u_{2} u_{3} v_{1} u_{4}=0 .
$$

Dos produtos de quatro elementos de $U$ e três elementos de $V$ resta provar que

$$
u_{1} \mathbf{v}_{3} u_{2} u_{3} u_{4}=0 \quad \text { e } \quad u_{1} u_{2} u_{3} \mathbf{v}_{3} u_{4}=0 .
$$

Segue do Lema 4.6 e das identidades acima que

$$
\begin{aligned}
u_{1} \mathbf{v}_{3} u_{2} u_{3} u_{4} & \stackrel{4.1 .4}{=} u_{3} \mathbf{v}_{3}\left(u_{1} u_{2}\right) u_{4} \\
& \stackrel{1.2 .7}{=}-u_{3} \mathbf{v}_{2}\left(u_{1} u_{2}\right) v_{3} u_{4} \\
& \stackrel{1.2 .6}{=} u_{3} \mathbf{v}_{2} u_{1} u_{2} v_{3} u_{4}+u_{3} \mathbf{v}_{2} u_{2} u_{1} v_{3} u_{4} \stackrel{4.1 .10}{=} 0 .
\end{aligned}
$$

E, por fim, temos

$$
\begin{aligned}
u_{1} u_{2} u_{3} \mathbf{v}_{3} u_{4} \stackrel{1.2 .7}{=}-u_{3} v_{1}\left(u_{1} u_{2}\right) v_{2} v_{3} u_{4} \\
\stackrel{1.2 .6}{=} u_{3} v_{1} u_{1} u_{2} v_{2} v_{3} u_{4}+u_{3} v_{1} u_{2} u_{1} v_{2} v_{3} u_{4} \stackrel{4.1 .10}{=} 0 .
\end{aligned}
$$

Lema 4.12 Sejam $A$ uma álgebra de Bernstein-Jordan, $u_{1}, u_{2}, u_{3} \in U$ e $v_{1} \in V$. Então

$$
\left(u_{1} v_{1} u_{2}^{2} u_{3}^{2}\right) N=0
$$


Demonstração. Pelo Lema 4.3 temos que $u_{1} v_{1} u_{2}^{2} u_{3}^{2} u=u v_{1} u_{2}^{2} u_{3}^{2} u_{1}$ e assim, podemos assumir, sem perda de generalidade, que $u=u_{1}$. Então segue que

$$
\begin{aligned}
u_{1} v_{1} u_{2}^{2} u_{3}^{2} u_{1} & \stackrel{1.2 .7}{=}-u_{1} u_{2}^{2} v_{1} u_{3}^{2} u_{1} \stackrel{4.6}{=}-u_{2} u_{1}^{2} v_{1} u_{3}^{2} u_{2} \\
& \stackrel{1.2 .7}{=}-u_{2} u_{3}^{2} u_{1}^{2} v_{1} u_{2} \stackrel{4.6}{=}-u_{3} u_{2}^{2} u_{1}^{2} v_{1} u_{3} \\
& \stackrel{1.2 .7}{=} u_{3} u_{1}^{2} u_{2}^{2} v_{1} u_{3} \stackrel{4.6}{=} u_{1} u_{3}^{2} u_{2}^{2} v_{1} u_{1} \\
& \stackrel{1.2 .7}{=}-u_{1} u_{3}^{2} v_{1} u_{2}^{2} u_{1} \stackrel{1.2 .7}{=} u_{1} v_{1} u_{3}^{2} u_{2}^{2} u_{1} \\
= & -u_{1} v_{1} u_{2}^{2} u_{3}^{2} u_{1},
\end{aligned}
$$

e assim, $2 u_{1} v_{1} u_{2}^{2} u_{3}^{2} u_{1}=0$.

Por outro lado, temos que

$$
\begin{aligned}
u_{1} v_{1} u_{2}^{2} u_{3}^{2} v & =-u_{1} v v_{1} u_{2}^{2} u_{3}^{2}=-u_{1} v v_{1} u_{2}^{2}\left(u_{3} u_{3}\right) \\
& \stackrel{4.6}{=}-u_{3} v v_{1} u_{2}^{2} u_{3} u_{1} \stackrel{1.2 .7}{=}-u_{3} u_{2}^{2} v v_{1} u_{3} u_{1} \\
& \stackrel{4.6}{=}-u_{2} u_{3}^{2} v v_{1} u_{2} u_{1} \stackrel{4.6}{=}-u_{1} u_{3}^{2} v v_{1} u_{2}^{2} \\
& \stackrel{1.2 .7}{=} u_{1} v v_{1} u_{2}^{2} u_{3}^{2}=-u_{1} v_{1} u_{2}^{2} u_{3}^{2} v
\end{aligned}
$$

e $\operatorname{assim} 2 u_{1} v_{1} u_{2}^{2} u_{3}^{2} v=0$, o que prova o lema.

Lema 4.13 Sejam A uma álgebra de Bernstein-Jordan, $u_{1}, u_{2}, u_{3} \in U e$ $v_{1}, v_{2} \in V$. Então

$$
\left(u_{1} v_{1} u_{2}^{2} v_{2} u_{3}\right) N=0 .
$$

Demonstração. Aplicando os Lemas 4.6 e 4.12, temos

$$
u_{1} v_{1} u_{2}^{2} v_{2} u_{3} u \stackrel{4.6}{=} u v_{1} u_{2}^{2} v_{2}\left(u_{1} u_{3}\right)=-u v_{1} u_{2}^{2}\left(u_{1} u_{3}\right) v_{2} \stackrel{4.12}{=} 0 .
$$

Agora, como $u_{1} v_{1} u_{2}^{2} v_{2} u_{3} \in V, \quad u_{1} v_{1} u_{2}^{2} v_{2} u_{3} v=0$, para todo $v \in V$ e assim, $\left(u_{1} v_{1} u_{2}^{2} v_{2} u_{3}\right) N=0$. 
Teorema 4.14 O barideal de uma álgebra de Bernstein-Jordan satisfaz

$$
\left(N^{2}\right)^{3} N=0
$$

Demonstração. Observamos que $N^{2}=U V+U^{2}$ e assim

$$
N^{2} N^{2}=U V U^{2}+(U V)(U V) \text { e } \quad\left(N^{2}\right)^{3}=U V U^{2} U^{2}+U V U^{2}(U V)
$$

logo segue dos Lemas 4.12 e 4.13 que

$$
\left(N^{2}\right)^{3} N=U V U^{2} U^{2} N+U V U^{2} V U N=0
$$

e o resultado está provado.

Corolário 4.15 A seguinte identidade é válida numa álgebra de BernsteinJordan

$$
\left(N^{2}\right)^{4}=0
$$

Demonstração. Aplicando o teorema anterior e o fato de $N^{2} \subset N$ temos

$$
\left(N^{2}\right)^{4}=\left(N^{2}\right)^{3} N^{2} \subset\left(N^{2}\right)^{3} N=0 .
$$

Seja agora $A$ uma álgebra de Bernstein. Pelo Corolário 1.11 temos que $A / L$ é uma álgebra de Bernstein-Jordan já que $V_{e}^{2} \subset L$ para cada idempotente $e$ de $A$. Seja $p\left(x_{1}, \cdots, x_{m}, z_{1}, \cdots, z_{t}\right)=0$ uma identidade para as álgebras de Bernstein-Jordan, isto é, para cada álgebra $B$ de BernsteinJordan, e para cada $\bar{f} \in I d A$ cumpre-se

$$
p\left(\bar{u}_{1}, \cdots, \bar{u}_{m}, \bar{v}_{1}, \cdots, \bar{v}_{t}\right)=0
$$


para todo $\bar{u}_{1}, \cdots, \bar{u}_{m} \in U_{\bar{f}}$ e $\bar{v}_{1}, \cdots, \bar{v}_{t} \in V_{\bar{f}}$. Já que $A / L$ é uma álgebra de Bernstein-Jordan temos então que $p$ também é uma identidade para $A / L$, e isto significa que para cada idempotente $e \in I d A$ temos

$$
p\left(u_{1}, \cdots, u_{m}, v_{1}, \cdots, v_{t}\right) \in L
$$

para todo $u_{1}, \cdots, u_{m} \in U_{e}$ e $v_{1}, \cdots, v_{t} \in V_{e}$. Tendo em conta que $L=$ $U_{e} \cap \operatorname{ann}\left(U_{e}+U_{e}^{2}\right)$ segue-se que

$$
\begin{gathered}
p\left(u_{1}, \cdots, u_{m}, v_{1}, \cdots, v_{t}\right) U=0 \quad \text { e } \\
p\left(u_{1}, \cdots, u_{m}, v_{1}, \cdots, v_{t}\right) U^{2}=0
\end{gathered}
$$

para cada $u, u_{1}, \cdots, u_{m} \in U_{e}$ e $v_{1}, \cdots, v_{t} \in V_{e}$. Em particular, como $(u v) v=0$ é uma identidade nas álgebras de Bernstein-Jordan, temos que

$$
[(u v) v] u_{1}=0 \quad \text { e } \quad[(u v) v] u_{1}^{2}=0
$$

para todo $u, u_{1} \in U_{e}$ e $v \in V_{e}$.

Corolário 4.16 O barideal de uma álgebra de Bernstein safisfaz

$$
\left(N^{2}\right)^{5}=0
$$

Demonstração. Pelo Corolário 4.15 temos que $\left(N^{2}\right)^{4} \subset L$, logo

$$
\left(N^{2}\right)^{5} \subset L N^{2} \subset L\left(U+U^{2}\right)=0
$$

e o resultado está provado.

Teorema 4.17 Seja A uma álgebra de Bernstein. Então

$$
N^{[3]}=0 .
$$


Demonstração. De (1.1.12) temos que

$$
\begin{aligned}
N^{[3]}= & \left(N^{2}\right)^{2}\left(N^{2}\right)^{2} \\
= & (U V) U^{2} \cdot(U V) U^{2} \\
& +(U V)(U V) \cdot(U V)(U V) \\
& +(U V)(U V) \cdot(U V) U^{2} .
\end{aligned}
$$

Resta-nos então analisar três situações, como segue. Primeiro vamos mostrar que $(U V) U^{2} \cdot(U V) U^{2}=0$. Temos que

$$
(U V) U^{2} \cdot(U V) U^{2}=(U V) U^{2} U^{2}(U V)=U V U^{2} U^{2} V U=0
$$

pelo Lema 4.12.

Agora, mostraremos que $(U V)(U V) \cdot(U V)(U V)=0$. Observe primeiro que

$$
\begin{gathered}
\left(u_{1} v_{1}\right)\left(u_{2} v_{2}\right) \cdot\left(u_{3} v_{3}\right)\left(u_{4} v_{4}\right) \stackrel{1.1 .16}{=} u_{1} \mathbf{v}_{2} u_{2} \cdot\left(u_{3} v_{3}\right)\left(u_{4} v_{4}\right) \stackrel{1.1 .10}{=} \\
u_{1} \mathbf{v}_{2}\left(u_{3} v_{3}\right) \cdot u_{2}\left(u_{4} v_{4}\right)+u_{1} \mathbf{v}_{2}\left(u_{4} v_{4}\right) \cdot u_{2}\left(u_{3} v_{3}\right)= \\
u_{1} \mathbf{v} v_{3} u_{3} \cdot u_{2}\left(u_{4} v_{4}\right)+u_{1} v_{1} v_{2} v_{4} u_{4} \cdot u_{2}\left(u_{3} v_{3}\right) .
\end{gathered}
$$

Portanto, é suficiente mostrar que

$$
U V V V U \cdot U V U=0
$$

Vejamos que $u_{1} \mathbf{v}_{3} u_{3} \cdot u_{2}\left(u_{4} v_{4}\right)=0$ para todo $u_{1}, u_{2}, u_{3}, u_{4} \in U$ e $v_{1}, v_{2}, v_{3}, v_{4} \in V$. Pelo Lema 4.6 podemos assumir, sem perda de generali- 
dade, que $u_{1}=u_{3}=x \in U$. Agora,

$$
\begin{aligned}
x \mathbf{v}_{\mathbf{3}} x \cdot u_{2}\left(u_{4} v_{4}\right) & =-x \mathbf{v}_{\mathbf{3}} u_{2} \cdot x\left(u_{4} v_{4}\right)-x \mathbf{v}_{\mathbf{3}}\left(u_{4} v_{4}\right) \cdot x u_{2} \\
& =-u_{2} \mathbf{v}_{\mathbf{3}} x \cdot\left(u_{4} v_{4}\right) x-u_{4} \mathbf{v}_{4} x \cdot u_{2} x \\
& =\frac{1}{2} u_{2} \mathbf{v}_{\mathbf{3}}\left(u_{4} v_{4}\right) x^{2}+\frac{1}{2} u_{4} \mathbf{v}_{\mathbf{4}} u_{2} x^{2} \\
& =-\frac{1}{2} u_{2} \mathbf{v}_{4} u_{4} x^{2}+\frac{1}{2} u_{4} \mathbf{v}_{4} u_{2} x^{2} \\
& =-u_{2} \mathbf{v}_{4} u_{4} x^{2} .
\end{aligned}
$$

Trocando $u_{2}$ com $u_{4}$ temos que $0=-2 u_{2} \mathbf{v}_{4} u_{4} x^{2}$, e a igualdade está provada.

Provaremos finalmente que $(U V)(U V) \cdot(U V) U^{2}=0$. Observe primeiro que

$$
\begin{aligned}
\left(u_{1} v_{1}\right)\left(u_{2} v_{2}\right) \cdot\left(u_{3} v_{3}\right)\left(u_{4} u_{5}\right)= & -\left(u_{3} v_{3}\right)\left(u_{4} u_{5}\right)\left(u_{1} v_{1}\right)\left(u_{2} v_{2}\right) \\
& -\left(u_{3} v_{3}\right)\left(u_{4} u_{5}\right)\left(u_{2} v_{2}\right)\left(u_{1} v_{1}\right) .
\end{aligned}
$$

Assim, é suficiente mostrar que $(U V) U^{2}(U V)(U V)=0$. Observe primeiro que $(U V) U^{2}(U V)(U V)=U V U^{2} V U(U V)$ e por Lema 4.3 este espaço vetorial é gerado por todos os elementos da forma $u v_{1} u_{1}^{2} v_{2} u\left(u_{2} v_{3}\right)$ com $u, u_{1}, u_{2} \in U$ e $v_{1}, v_{2}, v_{3} \in V$. Pela identidade (1.1.10) temos que

$$
\begin{aligned}
u v_{1} u_{1}^{2} v_{2} u \cdot u_{2} v_{3} & =-u v_{1} u_{1}^{2} v_{2} u_{2} \cdot u v_{3}-u v_{1} u_{1}^{2} v_{2} v_{3} \cdot u u_{2} \\
& =-u v_{1} u_{1}^{2} v_{2} u_{2} \cdot u v_{3}+u v_{1} u_{1}^{2} v_{2} v_{3} u u_{2}+u v_{1} u_{1}^{2} v_{2} v_{3} u_{2} u \\
& =-u v_{1} u_{1}^{2} v_{2} u_{2} \cdot u v_{3}
\end{aligned}
$$

já que pela Proposição 4.11 segue que $U V U^{2} V V U U=0$. Assim

$$
u v_{1} u_{1}^{2} v_{2} u_{2} \cdot u_{3} v_{3}=-u v_{1} u_{1}^{2} v_{2} u_{3} \cdot u_{2} v_{3}
$$


para todo $u, u_{1}, u_{2}, u_{3} \in U$ e $v_{1}, v_{2}, v_{3} \in V$. Finalmente,

$$
\begin{aligned}
u v_{1} u_{1}^{2} v_{2} u \cdot u_{2} v_{3} & =-u v_{1} u_{1}^{2} v_{2} u_{2} \cdot u v_{3} \\
& \stackrel{4.3}{=}-u_{2} v_{1} u_{1}^{2} v_{2} u \cdot u v_{3} \stackrel{4.1 .11}{=} 0 .
\end{aligned}
$$

o que demonstra o último caso. 


\section{Referências Bibliográficas}

[1] Abraham, V.M. A note on train algebras. Proc. Edinburgh Math. Soc. (2) 20:53-58 (1976).

[2] Bernad, J., González, S. And Martínez, C. On Nilpotency of the barideal of a Bernstein algebra. Comm. in Algebra., (9) 25:2967-2985 (1997).

[3] Burgueño, C. And Mallol, C., Morphismes de Peirce et Orthogonalité Dans les Algèbres de Bernstein, Linear Algebra and its applications., 219:179-186 (1995).

[4] Cortés, T. And Montaner, F. On the structure of Bernstein algebras. J.London Math. Soc., (2) 51:41-52 (1995).

[5] Cortés, T. And Montaner, F. Low dimensional Bernstein-Jordan algebras. J.London Math. Soc., (2) 51:53-61 (1995).

[6] Costa, R. And Picanço, J. Invariance of dimension of p-subespaces in Bernstein algebras. Comm. in Algebra., (8) 27:4039-4055 (1999). 
[7] Etherington, I.M.H. Genetic algebras. Proc. Roy. Soc. Endinburgh, 59:242-258 (1939).

[8] Etherington, I.M.H. Special train algebras. Quart. J. Math. Oxford, Ser. (2) 12:1-8 (1941).

[9] Etherington, I.M.H. Commutative train algebras of ranks 2 and 3. J. London Math. Soc., 15:136-149, (1940). Corrigendum ibid. 20:238 (1945).

[10] Fernández, J.C.G. Tesis Doctoral. Universidad de Oviedo, Spain (1994).

[11] Gerstenhaber, M. And Myung, H.C., On comutative power associative nilalgebras of low dimension, Proc. Amer. Math. Soc., (1) 48:2932 (1975).

[12] Gonshor, H. Contributions to genetic algebras. Proc. Edinburgh Math. Soc., 17:289-298 n.2 (1971).

[13] Gonshor, H. Contributions to genetic algebras II. Proc. Edinburgh Math. Soc., 18:273-279 n.2 (1973).

[14] GonzÁlez, S. ANd Martínez, C. Idempotent elements in a Bernstein algebra. J. London Math. Soc., (2) 42:430-436 (1990).

[15] Grishkov, A.N. On the genetic property of Bernstein algebras. Soviet Math. Dolk., 35:489-492 (1988).

[16] Guzzo, H., The Peirce decomposition for commutative train algebras, Comm. in Algebra., 22:5745-5757 n.14 (1994). 
[17] Guzzo, H. AND Pilar, V., Train algebras of rank $n$ which are Bernstein or power-associative algebras, Nova J. Math. Game Theory Algebra., 6:103-112 no. 2-3 (1997).

[18] Hentzel, I.R. AND Peresi, L.A. Semi-prime Bernstein algebras. Arch.Math., 52:539-543 (1989).

[19] Hentzel, I.R., D.P.Jacobs, P.Sverchkov and Peresi, L.A. Solvability of the ideal of all weight zero elements in Bernstein algebras. Comm.in Algebras., vol.22, no. 9 pp.3265-3275 (1994).

[20] Holgate, P. Characterizations of genetic algebras. J. London Math. Soc., (2) 6:169-174 (1972).

[21] Holgate, P. Genetics algebras satisfying Bernstein's stationarity principle, J. London Math. Soc., (2) 9:621-624 (1975).

[22] Iкемото, L.S. Álgebras de Bernstein: resultados recentes. Dissertação de Mestrado, Instituto de Matemática e Estatística da Universidade de São Paulo, 1997.

[23] Lelis, M.L. Formas P-invariantes em álgebras de Bernstein. PhD thesis, Instituto de Matemática e Estatística da Universidade de São Paulo, 1996.

[24] LyuBich, Y.I. Bernstein algebras, Uspekhi Mat. Nauk 32, No. 6 261263 (1977).

[25] LyUBICH, Y.I. Basic concepts and theorems of evolutionary genetics for the populations, Russian Math. Surveys. (5) 26:51-123 (1977) 
[26] LYUBICH, Y.I. Matematical structures in population genetics, volume 22, Biomathematic. Springer-Verlag, 1983.

[27] MARTínez, C. Free nuclear algebras. Jornal of Algebra., 177 676-697 (1995).

[28] Oliveira, R.A. Sobre os P-subespaços em uma train álgebras de posto 3. PhD thesis, Instituto de Matemática e Estatística da Universidade de São Paulo, 1997.

[29] Peresi, L.A. Nilpotency in Bernstein algebras. Arch.Math., 56:437-439 (1991).

[30] PiCANÇO, J. Subespaços Invariantes em algumas álgebras Báricas. PhD thesis, Instituto de Matemática e Estatística da Universidade de São Paulo, 1998.

[31] Quattara, M. Sur les Algèbres de Bernstein qui sont des T-Algèbres. Linear Algebra and its applications, 148:171-178 (1991).

[32] Reed, M.L. Algebras Structures of Genetic Inheritance, Bull. Amer. Math. Soc., (New Series), 34:107-131 n.2 (1997).

[33] Schafer, R.D. Structure of genetic algebras. Amer. J. Math., 71:121135 (1949).

[34] WALChER, S. Bernstein algebras which are Jordan algebras. Arch.Math., 50:218-222 (1988).

[35] Wörz-Busekros, A. Algebras in Genetics, volume 36, Lecture Notes in Biomathematics. Springer-Verlag,Berlin/New York, 1980. 
[36] Wörz-Busekros, A. Bernstein algebras. Arch.Math., 48:388-398 (1987). 


\section{Índice Remissivo}

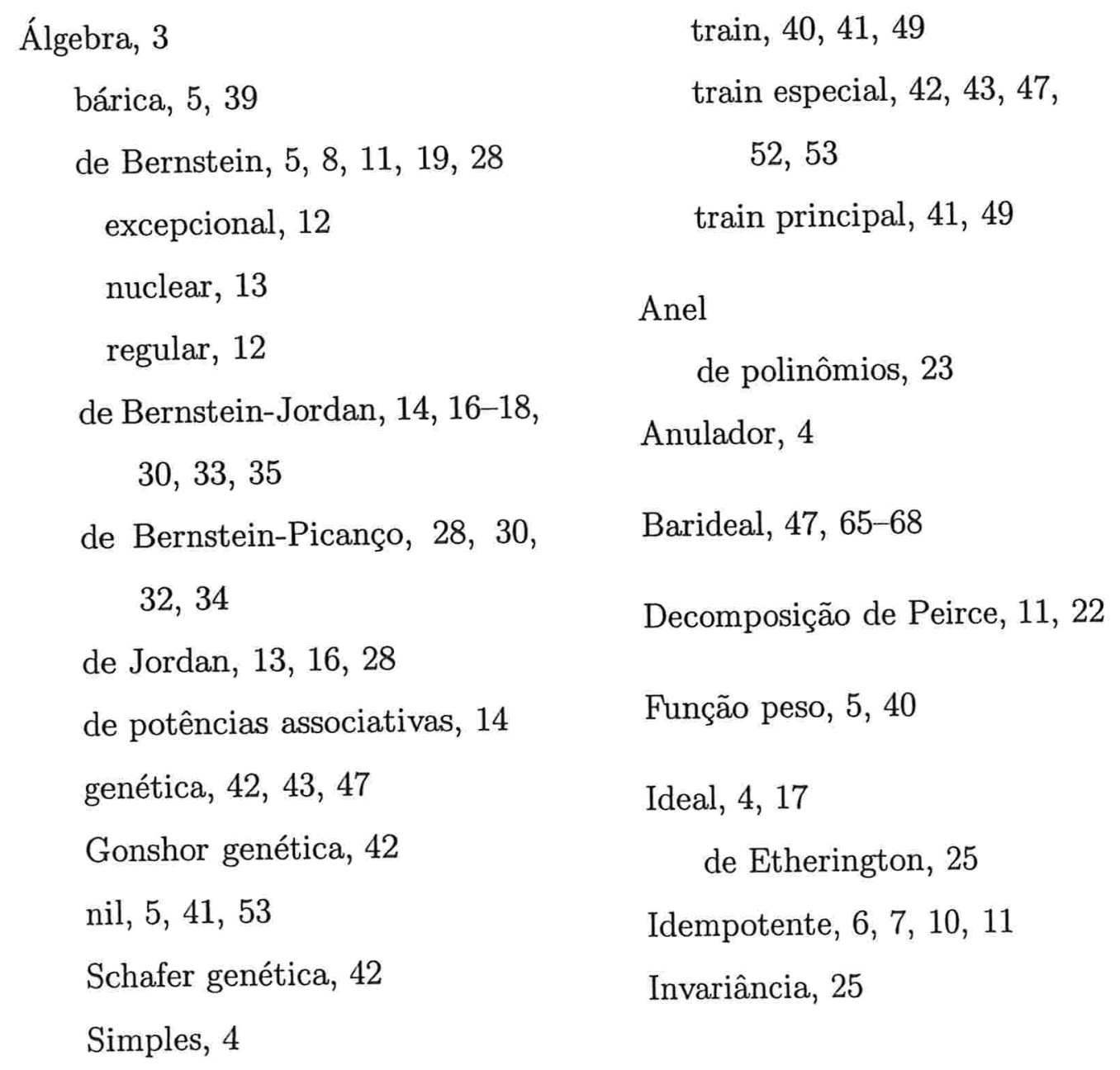


da dimensão dos P-subespaços,

$$
26,34
$$

dos P-subespaços, 22, 26, 34,

35

Isomorfismos, 11, 27, 28

Monômio

ímpar, 23

par, 23

Nilpotência, 5, 41, 47, 52, 53

P-subespaços, 19, 23

Polinômio

ímpar, 23

característico, 40, 42

minimal, 40

minimal principal, 41

par, 23

train, 39

Potências, 5

plenas, 5

principais, 4, 14, 43, 47

Produto

antisimétrico, 56

nulo, 62, 63

simétrico, 56
Raízes

$$
\text { train, 40, } 49
$$

Solubilidade, 67

Subespaço

$$
\begin{aligned}
& \text { monomial, } 22 \\
& \text { polinomial, } 22
\end{aligned}
$$

Tipo, 11 